\title{
Compressed-Sensing Assisted Spatial Multiplexing Aided Spatial Modulation
}

\author{
IEEE Transactions on Wireless Communications, 2017 \\ Lixia Xiao, Yue Xiao, Member, IEEE, Chao Xu, Xia Lei, Member, IEEE, Ping Yang, Senior Member, \\ IEEE, Shaoqian Li, Fellow, IEEE, and Lajos Hanzo, Fellow, IEEE
}

\begin{abstract}
Spatial-Multiplexing aided Spatial Modulation (SMx-SM) is proposed, which intrinsically amalgamates the concept of Vertical Bell Labs Space-Time (V-BLAST) and Spatial Modulation (SM) to attain a high transmission rate, despite its low number of Radio Frequency (RF) chains at the transmitter. Specifically, in the SMx-SM scheme, the Transmit Antennas (TAs) are partitioned into groups and the SM technique is applied individually to each group. Furthermore, lowcomplexity threshold-aided Compressive Sensing (CS) based and Message Passing (MP) based detectors are derived for our SMx-SM system. Our simulation results show that the proposed SMx-SM system exhibits a better performance despite its lower complexity than the Conventional Generalized Spatial Modulation (CGSM) system. More importantly, the proposed SMxSM system is capable of providing considerable performance gains over the V-BLAST system at the same number of RF chains and throughput. Finally, an upper bound is derived for the Average Bit Error Probability (ABEP), which is confirmed by our simulation results.
\end{abstract}

Index Terms - Spatial modulation (SM), MultipleInput Multiple-Output (MIMO), Vertical Bell Labs Space-Time (VBLAST).

\section{INTRODUCTION}

$\mathbf{L}$ ARGE-SCALE Multiple-Input Multiple-Output (MIMO) techniques [1], which are capable of dramatically improving the attainable transmit rate and transmit reliability by employing a multiplicity of transmit/receive antennas, have been considered as one of the key techniques in the next generation communication networks [2]-[3]. In massive MIMO systems, conventional MIMO solutions (e.g., VBLAST and STBC [4] [5]) require numerous radio frequency (RF) chains, hence imposing substantial implementation cost and signal processing complexity [1]. Spatial Modulation (SM) [6]-[8], which relies on the activated antenna indices as an additional means of implicitly conveying information, is a novel

Y. Xiao, L. Xiao, X. Lei, P. Yang, S. Li are with the National Key Laboratory of Science and Technology on Communications, University of Electronic Science and Technology of China 611731, Sichuan, China.

L. Xiao thanks to the National Science Foundation of China under Grant number 61471090 and 61501095, the open research fund of National Mobile Communications Research Laboratory, Southeast University (No. 2015D09).

$\mathrm{X}$. Chao and L. Hanzo are with the school of Electronics and Computer Science, University of Southampton, Southampton SO17 1BJ, U.K. (email: lh@ecs.soton.ac.uk).

L. Hanzo would like to thank the ERC for the Advanced Fellow Grant Beam-Me-Up. low-cost single RF chain-based MIMO transmission technique. Due to these advantages, SM has been shown to be a promising large-scale MIMO technique [7]-[13].

In SM scheme, only a single Transmit Antenna (TA) is activated to transmit one constellation symbol. In order to further increase the throughput of SM systems, Generalized SM (GSM) schemes were developed in [14][18], where more than one but not all TAs are activated to convey information symbols. Specifically, $N_{u}$ out of $N_{t}$ TAs are selected to transmit $N_{u}$ different symbols [16][18] for increasing the associated spectral efficiency, while maintaining a high energy efficiency, since in contrast to conventional MIMO systems (e.g., VBLAST and STBC [4] [5]) only $N_{u}$ out of $N_{t}$ RF chains are needed.

For the GSM scheme, the detection technique is more complicated than for SM schemes, as exemplified by the optimal Maximum Likelihood (ML) detector of [16]. Fortunately, a variety of low-complexity detectors have also been developed for the GSM scheme in [17]-[27]. More specifically, low-complexity Compressive Sensing (CS) based detectors are developed in [23]-[27], which exploit sparsity of GSM symbols. The Efficient CS (ECS) detectors of [27] are particularly attractive since they approach the ML detector's performance, despite their significantly reduced complexity in large-scale GSM systems, when using a small value of $N_{u}$. Additionally, a low-complexity near-optimal soft-decision based detector is proposed in [28]. On the other hand, the issues of broadband channel detection, precoding, and signal constellation design are developed in [29]-[32]. Moreover, GSM systems have been studied both in the context of $5 \mathrm{G}$ multiuser and multi-cell communication systems [33] [34] as well as in millimeter-wave communications [35].

However, the aforementioned GSM systems are design based for low-throughput (small-scale GSM system or for large-scale GSM systems with a small fraction of activated TAs). Additionally, the achievable rate and capacity of GSM system are analyzed in [36] [37]. However, for largescale GSM system having a large number of activated TAs, the cardinality of activated Transmit Antenna Combination (TAC) set is large. Considering $N_{t}=128$ and $N_{u}=16$ for example, the size of the TAC set is in excess of $2^{66}$. In the conventional bit-to-symbol mapping of the GSM system, a specific TAC index is selected from $2^{66}$ at the transmitter, which is extremely difficult. Although a low-complexity bit-to symbol mapping method is proposed in [37], it still a challenge to demap the estimated 
index into 66 bits at the receiver, which is impractical in simulations. Additionally, other SM variants have also been investigated in [39]-[43]. But the SM variants of [39][42] are mainly designed for two RF chains and hence are associated with a low throughput. The TAC set size of the SM variant in [43] is $2^{N_{t}}-1$, which is extremely high in large-scale MIMO systems, where hundreds of TAs are employed at the transmitter.

Against the above background, the contributions of this paper are summarized as follows:

1) A novel low-complexity high spectral efficiency MIMO scheme namely Spatial-Multiplexing-aided $\mathrm{SM}(\mathrm{SMx}-\mathrm{SM})$ is proposed. A pair of mapping methods are proposed for the SMx-SM system conceived, which are easy to map and demap in the context of large-scale SMx-SM systems.

2) Then, a low-complexity Threshold-aided Compressive Sensing (T-CS) detector and Message Passing (MP) detector are proposed for the SMx-SM system. Specifically, the T-CS detector is more suitable for a large-scale SMx-SM system having a small number of activated TAs, which is capable of approaching ML detector's performance, despite its significantly reduced complexity. The MP detector is eminently suitable for the large-scale SMx-SM systems having a large number of activated TAs.

3) Furthermore, an upper bound of the Average Bit Error Probability (ABEP) of the proposed SMx-SM system is also derived and compared. The proposed SMx-SM scheme outperforms the Conventional GSM (C-GSM) scheme at a lower complexity. Explicitly, the proposed SMx-SM scheme employing a large number of TAs is capable of providing a $6 \mathrm{~dB}$ Signal to Noise Ratio (SNR) gain over the V-BLAST system having the same number of RF chains and the same throughput.

The remainder of this paper is organized as follows. Section II provides a rudimentary review of the conventional SM and GSM systems. In Section III, the proposed SMxSM system model and generalized mapping as well as demapping methods are introduced. In Section IV, our T-CS detector is proposed for the SMx-SM system, while a MP based detector is developed in Section V. Our theoretical analysis are presented in Section VI. Section VII portrays our simulation results. Finally, Section VIII concludes this paper.

Notation: $\|\cdot\|_{F}$ denotes the Frobenious norms of a matrix. $|\cdot|$ represents the magnitude of a complex quantity. $(\cdot)^{T}$ and $(\cdot)^{H}$ stand for the transpose and the Hermitian transpose of a vector/matrix, respectively. $A \backslash B$ denotes removing the set $B$ from the set $A$.

\section{Conventional SM And GSM Systems}

\section{A. Conventional SM System}

We consider a SM system with $N_{t}$ TAs and $N_{r}$ receive antennas communicating over flat Rayleigh fading channels. In the conventional SM scheme, the information bits are divided into two parts, $\log _{2}\left(N_{t}\right)$ bits are used to select an active TA index from the index group $\Lambda=\left\{1,2, \ldots, N_{t}\right\}$ and $\log _{2}(M)$ bits are mapped to an $M$-ary APM symbol $s$.
Based on the above mapping rule, the transmitted signal $\mathbf{x} \in \mathbb{C}^{N_{t} \times 1}$ can be represented as

$$
\mathbf{x}=[\underbrace{0, \ldots, 0}_{q-1}, s, \underbrace{0, \ldots, 0}_{N_{t}-q}]^{T},
$$

where $q \in \Lambda$ denotes the index of activate TAs.

\section{B. Conventional GSM}

In order to further improve the transmission rate of SM systems, the C-GSM employing multiple activated TAs is developed in [16]-[18], where $N_{u}$ out of $N_{t}\left(N_{u}<N_{t}\right)$ TAs are activated for data transmission, so that $N_{\text {all }}=$ $C_{N_{t}}^{N_{u}}$ legitimate TACs are available. Among them, $N=$ $2^{\left\lfloor\log _{2}\left(C_{N_{t}}^{N u}\right)\right\rfloor}$ TACs are randomly chosen for encoding the information bits, $C_{m}^{n}$ represents the binomial coefficient and $\lfloor\bullet\rfloor$ denotes the floor operation. In each time slot, a vector of $B$ information bits is divided into two parts. The first block of $B_{1}=\left\lfloor\log _{2}\left(C_{N_{t}}^{N_{u}}\right)\right\rfloor$ bits is used to select a TAC $I_{i}(i \in(1, N))$, and the second block of $B_{2}=N_{u} \log _{2}(M)$ bits is used for modulating $N_{u} M$-ary APM symbols. Hence, the GSM transmit vector can be expressed as follows

$$
\mathbf{x}_{\left(I_{i}, \mathbf{s}\right)}=\left[\ldots, 0, s_{i_{1}}, 0, \ldots, 0, s_{i_{2}}, 0 \ldots, 0, s_{i_{N_{u}}}, 0, \ldots\right]^{T},
$$

where $\left(i_{1}, \ldots, i_{N_{u}}\right)$ indicates the activated TA indices.

For a large-scale C-GSM system, both $C_{N_{t}}^{N_{u}}$ and $N$ increases dramatically as $N_{t}$ and $N_{u}$ increases, hence the mapping process becomes challenging. Although, the authors of [37] proposed a simplified mapping method, it remains a challenge to estimate the TAC index at the receiver.

\section{Proposed Spatial Multiplex-Aided SM SYSTEM}

In order to address the mapping and demapping issues of GSM systems, a low-complexity yet spectral efficient SMx-SM scheme is proposed in this section. Considering a MIMO system having $N_{t}$ TAs, $N_{r}$ receiver antennas and $N_{u}$ activated TAs, the $N_{t}$ TAs are split into $N_{u}$ groups and in each group a single TA is activated. Considering $N_{t}=4$ and $N_{u}=2$ for example, the proposed SMx-SM symbol set $\mathbb{X}_{4}^{2}=\left[\mathbf{x}_{1}, \mathbf{x}_{2}\right]$ is constructed of two SM symbols as seen below

$$
\mathbb{X}_{4}^{2}=\left\{\left[\begin{array}{c}
\underbrace{s_{1}}_{\mathbf{x}_{1}} \\
s_{2} \\
\underbrace{0}_{\mathbf{x}_{2}}
\end{array}\right],\left[\begin{array}{c}
0 \\
\underbrace{s_{1}}_{\mathbf{x}_{1}} \\
s_{2} \\
\underbrace{0}_{\mathbf{x}_{2}}
\end{array}\right],\left[\begin{array}{c}
s_{1} \\
\underbrace{0}_{\mathbf{x}_{1}} \\
0 \\
\underbrace{s_{2}}_{\mathbf{x}_{2}}
\end{array}\right],\left[\begin{array}{c}
0 \\
\underbrace{s_{1}}_{\mathbf{x}_{1}} \\
0 \\
\underbrace{s_{2}}_{\mathbf{x}_{2}}
\end{array}\right]\right\},
$$

where $s_{1}$ and $s_{2}$ are the constellation symbols.

\section{A. Generalized System Model of SMx-SM}

The generalized system model of the proposed SMx-SM is shown in Fig. 1. As seen from Fig. 1, the information bits of $B=\sum_{i=1}^{N_{u}} B_{i}$ are partitioned into $N_{u}\left(1 \leq N_{u} \leq N_{t} / 2\right)$ groups and then the SM mapping principle is employed for each group. Assuming that the $i$-th $\left(i=1, \ldots, N_{u}\right) \mathrm{SM}$ 


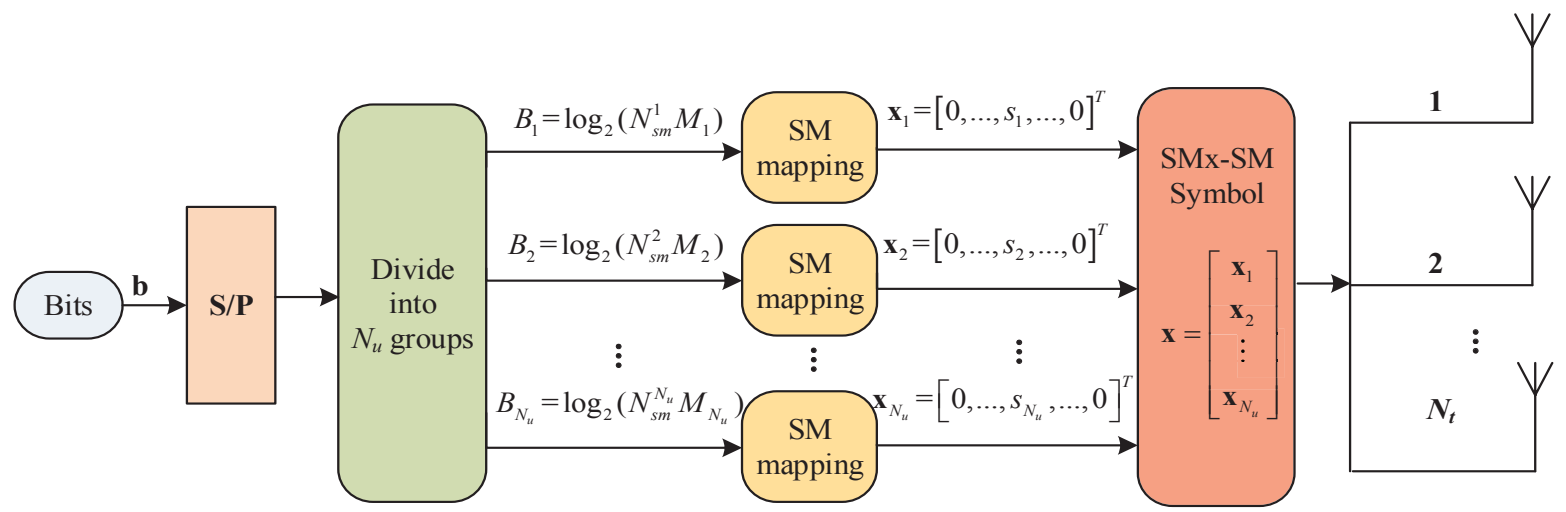

Fig. 1. System model of the proposed SMx-SM system

symbol employing $N_{s m}^{i}$ TAs and $M_{i}$ APM symbols, the $i$-th block of information bits having a length of $B_{i}=$ $\log _{2}\left(N_{s m}^{i}\right)+\log _{2}\left(M_{i}\right)$ can be mapped into a SM symbol as

$$
\mathbf{x}_{i}=[\underbrace{0, \ldots, 0}_{q_{i}-1}, s_{i}, \underbrace{0, \ldots, 0}_{N_{s m}^{i}-q_{i}}]^{T},
$$

where $q_{i}$ is the antenna index of $i$-th SM symbol. Hence, the transmitted signal can be expressed as

$$
\begin{array}{r}
\mathbf{x}=\left[\mathbf{x}_{1}^{T}, \mathbf{x}_{2}^{T}, \ldots, \mathbf{x}_{N_{u}}^{T}\right]^{T} \\
=[\underbrace{0, \ldots, 0}_{q_{1}-1}, s_{1}, \underbrace{0, \ldots, 0}_{N_{s m}^{1}-q_{1}}, \ldots, \underbrace{0, \ldots, 0}_{q_{N_{u}}-1}, s_{N_{u}}, \underbrace{0, \ldots, 0}_{\substack{N_{s m}^{N_{u}}-q_{N_{u}} \\
\uparrow}},]^{T}, \\
l_{1}=q_{1} \quad \begin{array}{l}
N_{N_{u}} \\
N_{u}^{N_{u}-1} N_{j=1}^{j} N_{s m}^{j}
\end{array}+q_{N_{u}}
\end{array}
$$

where $l_{i}\left(i=1, \ldots, N_{u}\right)$ denotes the location index for the $i$-th nonzero element in $\mathbf{x}$. Especially, when $M=1$ is employed, the SMx-SM symbol consists of $N_{u}$ Space Shift Keying (SSK) $[8]$ symbols as $\mathbf{x}=[0, \ldots, 0,1,0, \ldots 0,1,0, \ldots 0]$.

Let $\mathbf{H} \in \mathbb{C}^{N_{r} \times N_{t}}$ and $\mathbf{n} \in \mathbb{C}^{N_{r} \times 1}$ be the MIMO channel matrix and noise matrix, whose entries are complex-valued Gaussian distributed, yielding $\mathcal{C N}(0,1)$ and $\mathcal{C N}\left(0, \sigma^{2}\right)$, respectively. The received signal $\mathbf{y} \in \mathbb{C}^{N_{r} \times 1}$ can be formulated as

$$
\mathbf{y}=\mathbf{H x}+\mathbf{n}=\mathbf{H}_{I} \mathbf{s}+\mathbf{n},
$$

where $\mathbf{H}_{I}=\left(\mathbf{h}_{l_{1}}, \mathbf{h}_{l_{2}}, \ldots, \mathbf{h}_{l_{N_{u}}}\right)$ is the sub-matrix of $\mathbf{H}$ with $N_{u}$ columns, and $\mathbf{s}=\left(s_{1}, \ldots, s_{N_{u}}\right)^{T}$ is the transmit symbol vector corresponding to the TAC $I=\left(l_{1}, \ldots, l_{N_{u}}\right)$.

It follows from Eq. (6) that the optimal ML-based demodulator can be formulated as

$$
(\hat{I}, \hat{\mathbf{s}})_{\mathrm{ML}}=\underset{I \in \mathbb{I}, \mathbf{s} \in \mathbb{S}}{\arg \min }\left\|\mathbf{y}-\mathbf{H}_{I} \mathbf{s}\right\|_{F}^{2},
$$

where $\mathbb{I}$ is TAC set associated with size of $N_{P}=\prod_{i=1}^{N_{u}} N_{s m}^{i}$, and $\mathbb{S}$ is the set of $N_{u}$-element symbol vectors.

\section{B. Bit-to-Symbol Mapping and Symbol-to-Bit Demapping Principle}

1) Bit-to-Symbol Mapping: The transmit signal of the proposed SMx-SM system consists of $N_{u}$ SM symbols with each having a length of $N_{s m}^{1}, \ldots ., N_{s m}^{N_{u}}$. Hence the mapping process becomes straightforward for any value of $N_{t}$ and $N_{u}$. According to (5), determining the lengths of $N_{s m}^{1}, \ldots ., N_{s m}^{N_{u}}$ is the key issue of the bit-to-symbol

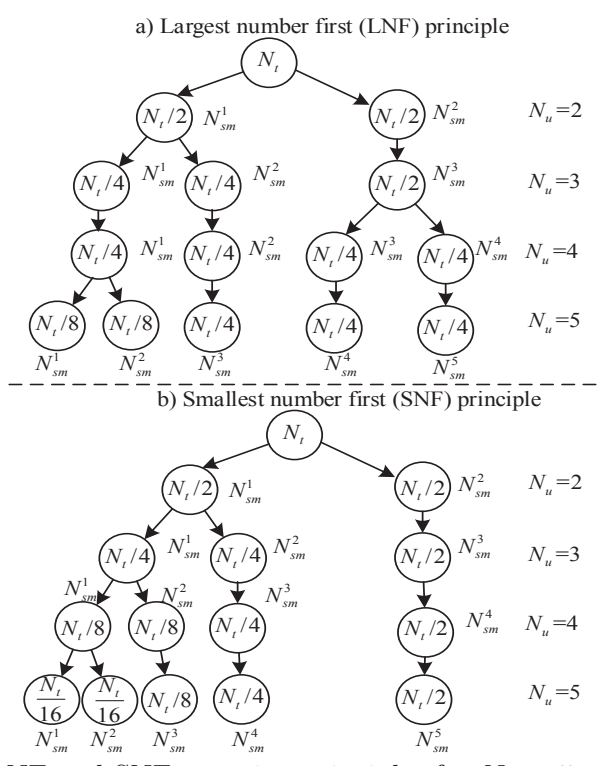

Fig. 2. LNF and SNF mapping principles for $N_{u}=5$

mapping. For simplicity, we introduce a pair of lowcomplexity methods based on the Largest Number First (LNF) principle and the Smallest Number First (SNF) principle to determine $N_{s m}^{1}, \ldots, N_{s m}^{N_{u}}$. Both the LNF and SNF techniques relying on $\left(N_{u}-1\right)$ steps and we consider $N_{s m}^{1} \leq N_{s m}^{2} \leq \ldots . \leq N_{s m}^{N_{u}}$ for simplicity.

LNF based Principle: In the $t$-th $t \in\left(2, \ldots, N_{u}-1\right)$ step, the largest number $N_{t-1}^{L}$ in the $(t-1)$-th set $\mathcal{N}_{t-1}$ is decomposed into two equal numbers as $\left(N_{t-1}^{L} / 2, N_{t-1}^{L} / 2\right)$, where $\mathcal{N}_{1}=\left[N_{t}\right]$. As a result, the decomposed set in the $t$-th step can be expressed as $\mathcal{N}_{t}=$ $\left(\mathcal{N}_{t-1} \backslash N_{t-1}^{L}, N_{t-1}^{L} / 2, N_{t-1}^{L} / 2\right)$. These processes continue, until we get $N_{u}$ values as $N_{s m}^{1}, \ldots ., N_{s m}^{N_{u}}$.

SNF based Principle: The main difference between the above-mentioned LNF based principle and its SNF based counterpart is that the smallest number $N_{t-1}^{S}>2$ in the $(t-1)$-th set $\mathcal{N}_{t-1}$ is decomposed into two equal number as $\left(N_{t-1}^{S} / 2, N_{t-1}^{S} / 2\right)$. This is because when the smallest number satisfies $N_{t-1}^{S} \leq 2$, the number $N_{t-1}^{S}$ can not decomposed into two equal number to map two SM symbols.

Fig. 2 presents an example for the case of $N_{u}=5$. The SMx-SM system associated with the LNF based and SNF based principles are the same for the case of $N_{u}=2$ and $N_{u}=3$, but they are different from each other for the case of $N_{t}>3$. For the case of $N_{u}=5$, the $N_{u} \mathrm{SM}$ 
symbols of the LNF based and SNF based principles are $\mathcal{N}_{5, \mathrm{LNF}}=\left[N_{t} / 8, N_{t} / 8, N_{t} / 4, N_{t} / 4, N_{t} / 4\right]$ and $\mathcal{N}_{5, \mathrm{SNF}}=$ $\left[N_{t} / 16, N_{t} / 16, N_{t} / 8, N_{t} / 4, N_{t} / 2\right]$, respectively. Moreover, each SM symbol has the length of $N_{s m}^{i} \geq 2$, hence we have $N_{u} \leq N_{t} / 2$ in the proposed SMx-SM system.

In order to make the SNF and LNF principles more explicit, Table I shows the size of the TAC set for the proposed SMx-SM system and for the C-GSM system associated with $N_{t}=32$. As seen from the Table I, the total number of the C-GSM system's TACs $N_{\text {all }}$ is larger than that of the SMx-SM system and increases significantly as $N_{t}$ and $N_{u}$ increases. Although selected $N$ randomly ${ }^{1}$ from $N_{\text {all }}$ ones, the selecting and mapping processes are still difficult and become impractical with large values of $N_{t}$ and $N_{u}$. For the proposed SMx-SM system, it only needs to map $N_{u}$ SM symbols having a length of $N_{s m}^{1}, \ldots, N_{s m}^{N_{u}}$, which is easy and straightforward for any numbers of $N_{t}$ and $N_{u}$.

2) Symbol-to-Bit Demapping: At the receiver, the TAC de-mapping processes of the LNF-based and SNF-based principle are the same, which rely on the values of $\left(N_{s m}^{1}, \ldots, N_{s m}^{N_{u}}\right)$ and the specific TAC of $I=\left(l_{1}, l_{2} \ldots, l_{N_{u}}\right)$. For our SMx-SM system, there are $N_{u}$ levels, each of which corresponds to one SM symbol $\left(l_{i}, s_{i}\right) i \in\left(1, \ldots, N_{u}\right)$. Based on the V-BLAST demapping method [4], $\left(l_{i}, s_{i}\right)$ can be demodulated into information bits as follows.

Step 1: Obtain the TA index for each SM symbol via $l_{i}$ and $(5)$ as $q_{i}=l_{i}-\sum_{j=1}^{i-1} N_{s m}^{j}$.

Step 2: Demodulate $q_{i}$ into information bits according to $\mathbf{b}_{i}^{T A C}=\operatorname{de} 2 \mathrm{bi}\left[q_{i}, \log _{2}\left(N_{s m}^{i}\right)\right]$, where $\operatorname{de} 2 \mathrm{bi}(m, n)$ is a function that transforms a decimal number $m$ to $n$ bits. Demodulation $s_{i}$ into information bits as $\mathbf{b}_{i}^{S y m}$ is based on [4]. Hence the $i$-th SM symbol is demodulated into bits as $\mathbf{b}_{i}=\left[\mathbf{b}_{i}^{T A C}, \mathbf{b}_{i}^{S y m}\right]$.

Step 3: Finally, we obtain the demodulated bits as $\mathbf{b}=$ $\left[\mathbf{b}_{1}, \ldots, \mathbf{b}_{i}, \ldots, \mathbf{b}_{N_{u}}\right]$.

Taking $N_{t}=8, N_{u}=3$ and BPSK for example, the bit-to-symbol mapping and symbol-to-bit demapping processes are introduced as follows. Firstly, we obtain the length of each SM symbol based on either the LNF or the SNF principle as $N_{s m}^{1}=2, N_{s m}^{2}=2$ and $N_{s m}^{3}=4$ in conjunction with $M_{1}=M_{2}=M_{3}=2$. Assuming that the information bits are $\mathbf{b}=[0110111]$, which can be partitioned into 3 groups as [01], [10], [111], we obtain three SM symbols as $\mathbf{x}_{1}=\left[\begin{array}{ll}1 & 0\end{array}\right]^{T}, \mathbf{x}_{2}=\left[\begin{array}{ll}0 & -1\end{array}\right]^{T}$ and $\mathbf{x}_{3}=\left[\begin{array}{llll}0 & 0 & 0 & 1\end{array}\right]^{T}$. Then we obtain the SMx-SM symbol as

$$
01,10,111 \rightarrow \mathbf{x}=\left[\begin{array}{llllllll}
1 & 0 & 0 & -1 & 0 & 0 & 0 & 1
\end{array}\right]^{T} .
$$

At the receiver, if the estimated TAC is $I=\left(l_{1}, l_{2}, l_{3}\right)=$ $(1,4,8)$ and the constellation symbol vector is $\hat{\mathbf{s}}=$ $\left[s_{1}, s_{2}, s_{3}\right]=[1.25,-0.9,1.1]$, the demodulation process is

\footnotetext{
${ }^{1}$ The authors of [17] employed different rotation angles to each constellation symbol and determined the optimal codeword set of the C-GSM system by maximizing the minimum distances between two different codewords. However, the optimal set selection technique of [17] is more suitable for small values of $N_{t}$ and $N_{u}$. This is because $C_{N_{\text {all }}}^{N}$ number of minimum distances have to be calculated. Observe from Table I that this becomes impractical for large values of $N_{t}$ and $N_{u}$. For simplicity, most research on C-GSM was dedicated to selecting $N$ TACs randomly.
}

expressed as

$$
\begin{aligned}
& \left(l_{1}, s_{1}\right) \rightarrow(01): l_{1}=1 \rightarrow q_{1}=1 \rightarrow 0, s_{1}=1.25 \rightarrow 1 \\
& \left(l_{2}, s_{2}\right) \rightarrow(10): l_{2}=4 \rightarrow q_{2}=2 \rightarrow 1, s_{2}=-0.9 \rightarrow 0 \\
& \left(l_{3}, s_{3}\right) \rightarrow(111): l_{3}=8 \rightarrow q_{3}=4 \rightarrow 11, s_{2}=1.1 \rightarrow 1 .
\end{aligned}
$$

In summary, it is unproblematic for the proposed SMx-SM system to perform bit-to-symbol mapping at the transmitter and symbol-to-bit demapping at the receiver for largescale MIMO system, whilst it can not be implemented in the C-GSM system.

\section{Performance Advantages over the} CONVEntional GSM AND V-BLAST SYSTEMS

In this section, the advantages of the proposed SMxSM system over the C-GSM and V-BLAST systems are analyzed in terms of its ABEP theoretical results.

\section{A. The ABEP of the proposed SMx-SM system}

We denote by $\mathbf{x}^{i}$ and $\mathbf{x}^{j}$ the SMx-SM transmit signal and receive signal, respectively. The ABEP upper bound is given by

$$
P_{b}=\frac{1}{B 2^{B}} \sum_{i=1}^{2^{B}} \sum_{j=1, j \neq i}^{2^{B}} d\left(\mathbf{x}^{i}, \mathbf{x}^{j}\right) P\left(\mathbf{x}^{i} \rightarrow \mathbf{x}^{j}\right),
$$

where $P\left(\mathbf{x}^{i} \rightarrow \mathbf{x}^{j}\right)$ denotes the pairwise error probability (PEP), $d\left(\mathbf{x}^{i}, \mathbf{x}^{j}\right)$ is the Hamming Distance (HD) associated with the corresponding PEP event. Based on [27], the PEP is expressed as

$$
P\left(\mathbf{x}^{i} \rightarrow \mathbf{x}^{j} \mid \mathbf{H}\right)=Q\left(\sqrt{\frac{\left\|\mathbf{H}\left(\mathbf{x}^{i}-\mathbf{x}^{j}\right)\right\|^{2}}{2 \sigma^{2}}}\right)=Q(\sqrt{\zeta}),
$$

where $Q(x)=(1 / \sqrt{2 \pi}) \int_{x}^{\infty} e^{-t^{2} / 2} d_{t}$ and $\zeta=\frac{\left\|\mathbf{H}\left(\mathbf{x}^{i}-\mathbf{x}^{j}\right)\right\|^{2}}{2 \sigma^{2}}$. As shown in [39], the average PEP can be written as

$$
P\left(\mathbf{x}^{i} \rightarrow \mathbf{x}^{j}\right)=\gamma(\bar{\zeta}) \sum_{k=0}^{N_{r}-1}\left(\begin{array}{c}
N_{r}-1+k \\
k
\end{array}\right)[1-\gamma(\bar{\zeta})]^{k}
$$

where $\gamma(\bar{\zeta})=\frac{1}{2}\left(1-\sqrt{\frac{\bar{\zeta} / 2}{1+\bar{\zeta} / 2}}\right)$ and $\bar{\zeta}$ is the mean value of $\zeta$ with $N_{r}=1$.

Assuming that the Antenna Indices (AIs) of the transmit signal $\mathbf{x}^{i}=\left[\mathbf{x}_{1}^{i}, \ldots, \mathbf{x}_{N_{u}}^{i}\right]$ and estimated signal $\mathbf{x}^{j}=$ $\left[\mathbf{x}_{1}^{j}, \ldots, \mathbf{x}_{N_{u}}^{j}\right]$ are $\left(l_{1}, l_{2}, \ldots, l_{N_{u}}\right)$ and $\left(\hat{l}_{1}, \hat{l}_{2}, \ldots, \hat{l}_{N_{u}}\right)$, respectively, and the corresponding symbol vectors are $\mathbf{s}=$ $\left[s_{1}, s_{2}, \ldots, s_{N_{u}}\right]^{T}$ and $\hat{\mathbf{s}}=\left[\hat{s}_{1}, \hat{s}_{2}, \ldots, \hat{s}_{N_{u}}\right]^{T}$, the value of $\vec{\zeta}$ for the $\mathrm{SMx}-\mathrm{SM}$ is given by

$$
\begin{aligned}
& \bar{\zeta}=E\left(\left(\left\|\mathbf{H}\left(\mathbf{x}^{i}-\mathbf{x}^{j}\right)\right\|^{2} / 2 \sigma^{2}\right) \mid N_{r}=1\right) \\
& =\left(\mathbf{x}^{i}-\mathbf{x}^{j}\right)^{H} E\left(\mathbf{H}^{H} \mathbf{H} \mid N_{r}=1\right)\left(\mathbf{x}^{i}-\mathbf{x}^{j}\right) / 2 \sigma^{2} \\
& =\left\|\left(\mathbf{x}^{i}-\mathbf{x}^{j}\right)\right\|^{2} / 2 \sigma^{2} \\
& =\sum_{t=1}^{N_{u}}\left\|\left(\mathbf{x}_{t}^{i}-\mathbf{x}_{t}^{j}\right)\right\|^{2} / 2 \sigma^{2} \\
& =\left\{\begin{array}{l}
\frac{\left|s_{1}-\hat{s}_{1}\right|^{2}+\cdots+\left|s_{N_{u}}-\hat{s}_{N_{u}}\right|^{2}}{2 \sigma^{2}}, \text { if } n=N_{u} \\
\frac{\left|s_{1}-\hat{s}_{1}\right|^{2}+\cdots+\left|s_{n}-\hat{s}_{n}\right|^{2}+2\left(N_{u}-n\right)}{2 \sigma^{2}}, \text { if } 0<n<N_{u} \\
\frac{2 N_{u}}{2 \sigma^{2}}, \text { if } n=0,
\end{array},\right.
\end{aligned}
$$

where $n$ is the number of identical AIs between $\left(l_{1}, l_{2}, \ldots, l_{N_{u}}\right)$ and $\left(\hat{l}_{1}, \hat{l}_{2}, \ldots, \hat{l}_{N_{u}}\right)$. Based on the values $\bar{\zeta}$ obtained from Eq. (13), the ABEP of the SMx-SM system can be expressed from (10) and (12). 
TABLE I

TAC MAPping OF THE PROPOSED SMX-SM with $N_{t}=32$.

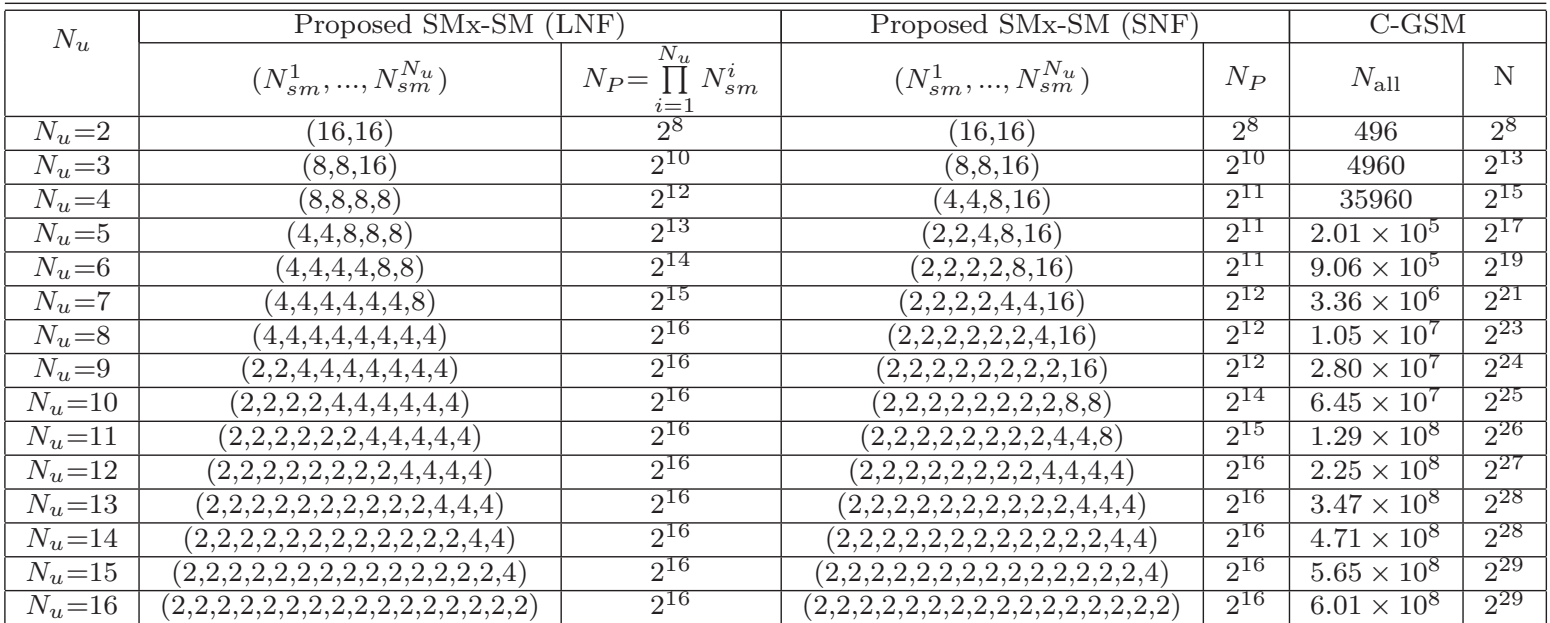

\section{B. Simplified ABEP Expressions}

From the above analysis, we find that for the calculation of the final ABEP we have to compute $2^{2 B} \mathrm{HD}$ values of $d\left(\mathbf{x}^{i}, \mathbf{x}^{j}\right)$ and $\bar{\zeta}$ in (13), which becomes impractical for a high transmit rate $B$. Observe from (13) that there are lots of identical values of $\bar{\zeta}$ and the number of different values of $\bar{\zeta}$ is finite for a fixed constellation size $M, N_{r}$ and SNR variance $\sigma^{2}$. Hence, the above ABEP expressions can be further simplified. Firstly, let us define $P\left(\mathbf{x}^{i} \rightarrow \mathbf{x}^{j}\right)$ as a function of $\bar{\zeta}$ in (13) as

$$
F(\bar{\zeta})=\gamma(\bar{\zeta}) \sum_{k=0}^{N_{r}-1}\left(\begin{array}{c}
N_{r}-1+k \\
k
\end{array}\right)[1-\gamma(\bar{\zeta})]^{k} .
$$

Assuming that the SMx-SM systems have $m$ different values $\bar{\zeta}$ as $\bar{\zeta}_{1}, \ldots, \bar{\zeta}_{m}$, the corresponding PEP values $F\left(\bar{\varsigma}_{1}\right), \ldots, F\left(\bar{\zeta}_{m}\right)$ can be obtained by (14), so that (10) can be represented as

$$
\begin{aligned}
& P_{b}=\frac{\sum_{t=1}^{\lambda_{1}} d d_{\bar{\varsigma}_{1}}^{t} F\left(\bar{\varsigma}_{1}\right)+\sum_{t=1}^{\lambda_{2}} d_{\bar{\varsigma}_{2}}^{t} F\left(\bar{\varsigma}_{2}\right)+\cdots+\sum_{t=1}^{\lambda} d_{\bar{\varsigma}_{2}}^{t} F\left(\bar{\varsigma}_{m}\right)}{=D\left(\bar{\varsigma}_{1}\right) F\left(\bar{\varsigma}_{1}\right)+D\left(\bar{\varsigma}_{m}\right) F\left(\bar{\varsigma}_{m}\right)+\cdots+D\left(\bar{\varsigma}_{m}\right) F\left(\bar{\varsigma}_{m}\right),} \\
& =D
\end{aligned}
$$

with

$$
\begin{gathered}
D\left(\bar{\varsigma}_{p}\right)=\sum_{t=1}^{\lambda_{p}} d_{\bar{\zeta}_{p}}^{t} / B 2^{B}, p=1, \ldots, m \\
\sum_{t=1}^{\lambda_{1}} d_{\bar{\zeta}_{1}}^{t}+\cdots+\sum_{t=1}^{\lambda_{m}} d_{\bar{\varsigma}_{m}}^{t}=\sum_{i=1}^{2^{B}} \sum_{j=1}^{2^{B}} d\left(\mathbf{x}^{i}, x^{j}\right)=2^{B} \sum_{u=1}^{B} C_{B}^{u} u
\end{gathered}
$$

where $\lambda_{p} p \in\{1, \ldots, m\}$ is the total number of candidate values $\bar{\zeta}_{p}$ for all the $\mathbf{x}^{i}$ and $\mathbf{x}^{j}$, while $d_{\bar{\zeta}_{p}}^{t}$ is the corresponding $\mathrm{HD}$, and $D\left(\bar{\zeta}_{p}\right)$ is the Average $\mathrm{HD}$ (AHD) for the value $\bar{\zeta}_{p}$. Moreover, for the proposed SMx-SM system, due to the symmetry of the system, we have $\sum_{t=1}^{\lambda_{p}} d_{\bar{\varsigma}_{p}}^{t}=2^{B} \sum_{t=1}^{\varphi_{p}} d_{\bar{\varsigma}_{p}}^{t}$, where $\varphi_{p}$ is the number of $\bar{\varsigma}_{p}$ for $\left(\mathbf{x}^{1}, \mathbf{x}^{j}\right)$. The ABEP performance of the SMx-SM system can be finally simplified to

$$
\begin{gathered}
P_{b}=\frac{\sum_{t=1}^{\varphi_{1}} d_{\bar{\varsigma}_{1}}^{t} F\left(\bar{\varsigma}_{1}\right)+\sum_{t=1}^{\varphi_{2}} d_{\bar{\varsigma}_{2}}^{t} F\left(\bar{\varsigma}_{2}\right)+\cdots+\sum_{t=1}^{\varphi_{m}} d_{\bar{\varsigma}_{2}}^{t} F\left(\bar{\varsigma}_{m}\right)}{B}, \\
=D\left(\bar{\varsigma}_{1}\right) F\left(\bar{\varsigma}_{1}\right)+D\left(\bar{\varsigma}_{m}\right) F\left(\bar{\varsigma}_{m}\right)+\cdots+D\left(\bar{\varsigma}_{m}\right) F\left(\bar{\varsigma}_{m}\right)
\end{gathered}
$$

with

$$
\begin{aligned}
& D\left(\bar{\varsigma}_{p}\right)=\frac{\sum_{t=1}^{\varphi_{p}} d_{\bar{\zeta}_{p}}^{t}}{B}, p=1, \ldots, m \\
& \sum_{t=1}^{\varphi_{1}} d_{\bar{\varsigma}_{1}}^{t}+\cdots+\sum_{t=1}^{\varphi_{m}} d_{\bar{\varsigma}_{m}}^{t}=\sum_{j=1}^{2^{B}} d\left(\mathbf{x}^{1}, x^{j}\right)=\sum_{u=1}^{B} C_{B}^{u} u .
\end{aligned}
$$

As a result, the calculation of ABEP only has to find the $m$ different values of $\bar{\zeta}_{1}, \ldots, \bar{\zeta}_{m}$ and to compute their corresponding values of $F\left(\bar{\varsigma}_{1}\right), \ldots, F\left(\bar{\varsigma}_{m}\right)$ and $D\left(\bar{\varsigma}_{1}\right), \ldots, D\left(\bar{\varsigma}_{m}\right)$, which substantially simplifies the ABEP calculation.

\section{Performance Advantage over the Conventional GSM System}

In this subsection, the performance advantage of the proposed SMx-SM system over the C-GSM system is analyzed based on ABEP analysis. Observe from (13) that the SMx-SM system and C-GSM system have the same values of $\bar{\zeta}_{m}$ for the same antenna configurations, hence the main difference between them lies in their AHD values $D\left(\bar{\varsigma}_{1}\right), \ldots, D\left(\bar{\varsigma}_{m}\right)$. Next, the differences between $\mathrm{C}$ GSM and the proposed SMx-SM system are analyzed in detail as follows.

1) Case $1 N_{u}=2$ and $M=1$ : For the case of $N_{u}=2$ and $M=1$, there are only two different values $\bar{\zeta}$ in (13) as $\varsigma_{1}=1 / \sigma^{2}$ and $\varsigma_{2}=2 / \sigma^{2}$. Hence, (17) can be expressed as

$$
P_{b}=D\left(\bar{\varsigma}_{1}\right) F\left(\bar{\varsigma}_{1}\right)+D\left(\bar{\varsigma}_{2}\right) F\left(\bar{\varsigma}_{2}\right) .
$$

Assuming that the AHDs of $\varsigma_{1}=1 / \sigma^{2}$ and $\varsigma_{2}=2 / \sigma^{2}$ in C-GSM system are $D_{C}\left(\bar{\varsigma}_{1}\right), D_{C}\left(\bar{\varsigma}_{2}\right)$ and $P_{b}^{\mathrm{C}}$ is the ABEP of C-GSM system, according to (15) and (18), we have $D\left(\bar{\varsigma}_{1}\right)+D\left(\bar{\varsigma}_{2}\right)=D_{C}\left(\bar{\varsigma}_{1}\right)+D_{C}\left(\bar{\varsigma}_{2}\right)$ and

$$
\begin{aligned}
& P_{b}-P_{b}^{\mathrm{C}}=\left[D\left(\bar{\varsigma}_{1}\right) F\left(\bar{\varsigma}_{1}\right)+D\left(\bar{\varsigma}_{2}\right) F\left(\bar{\varsigma}_{2}\right)\right]- \\
& {\left[D_{C}\left(\bar{\varsigma}_{1}\right) F\left(\bar{\varsigma}_{1}\right)+D_{C}\left(\bar{\varsigma}_{2}\right) F\left(\bar{\varsigma}_{2}\right)\right]} \\
& =\left[F\left(\bar{\varsigma}_{2}\right)-F\left(\bar{\varsigma}_{1}\right)\right)\left(D_{C}\left(\bar{\varsigma}_{1}\right)-D\left(\bar{\varsigma}_{1}\right)\right] .
\end{aligned}
$$

Furthermore, the values of $F(\bar{\zeta})$ associated with different values of $\bar{\zeta}$ and $N_{r}$ are depicted in Fig. 2. It's evident in Fig. 2 that the value of $F(\bar{\zeta})$ decreases as $\bar{\zeta}$ and $N_{r}$ increases. In this case, since $1 / \sigma^{2}<2 / \sigma^{2}$, we have $F\left(\bar{\varsigma}_{1}\right)>$ $F\left(\bar{\varsigma}_{2}\right)$. As a result, $D\left(\bar{\varsigma}_{1}\right)$ and $D_{C}\left(\bar{\varsigma}_{1}\right)$ play an important role in the performance comparison between the SMx-SM system and C-GSM system. 
TABLE II

The average hamming distances of the $M=1$ aided SMX-SM and C-GSM systems having Different antenna Configurations

\begin{tabular}{|c|c|c|c|c|c|c|c|c|c|c|}
\hline & \multicolumn{3}{|c|}{$\overline{N_{t}=8}$} & \multicolumn{3}{|c|}{$N_{t}=16$} & \multicolumn{3}{|c|}{$N_{t}=32$} \\
\hline & & $\begin{array}{c}\text { SMx-SM } \\
\text { LNF }\end{array}$ & $\begin{array}{l}\text { SMx-SM } \\
\text { SNF }\end{array}$ & C-GSM & $\begin{array}{c}\text { SMx-SM } \\
\text { LNF }\end{array}$ & $\begin{array}{c}\text { SMx-SM } \\
\text { SNF }\end{array}$ & C-GSM & $\begin{array}{c}\text { SMx-SM } \\
\text { LNF }\end{array}$ & $\begin{array}{c}\text { SMx-SM } \\
\text { SNF }\end{array}$ & C-GSM \\
\hline \multirow[b]{2}{*}{$N_{u}=2$} & $D\left(\bar{\varsigma}_{1}\right)$ & 2 & 2 & 3.59 & 4 & 4 & 7.38 & 8 & 8 & 15.55 \\
\hline & $D\left(\bar{\varsigma}_{2}\right)$ & 6 & 6 & 4.40 & 28 & 28 & 24.62 & 120 & 120 & 112.46 \\
\hline \multirow{4}{*}{$N_{u}=4$} & $D\left(\bar{\varsigma}_{1}\right)$ & 1 & 1 & 7.40 & 2 & 2.57 & 13.47 & 4 & 4.73 & 51.02 \\
\hline & $D\left(\bar{\varsigma}_{2}\right)$ & 3 & 3 & 166 & 18 & 16.85 & 111.3 & 84 & 78.9 & 1033 \\
\hline & $D\left(\bar{\varsigma}_{3}\right)$ & 3 & 3 & 750 & 54 & 29.42 & 247 & 588 & 380.7 & 5970 \\
\hline & $D\left(\bar{\varsigma}_{4}\right)$ & 1 & 1 & 5 & 54 & 15.43 & 139.5 & 1372 & 559.6 & 9328 \\
\hline
\end{tabular}

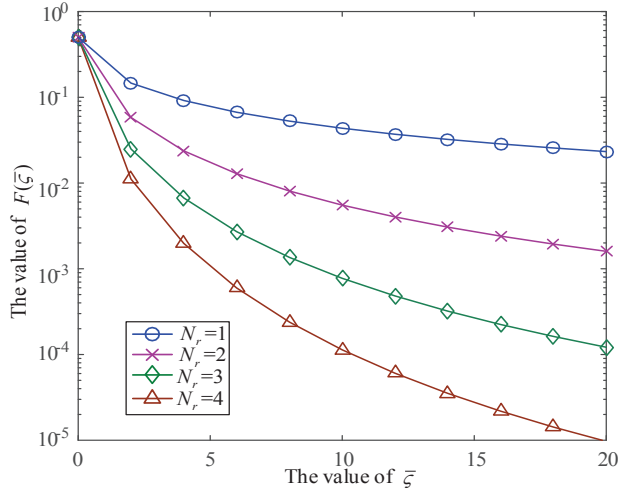

Fig. 3. The values of $F(\bar{\varsigma})$.

2) Other MIMO Setups: For the case of $N_{u}>2$ with the same modulation level, the transmit rate of the proposed SMx-SM system $B$ and that of the C-GSM system $B_{C}$ are different, while the values of $F\left(\bar{s}_{p}\right) p \in(1, \ldots, m)$ are the same. Hence, the performances of the SMx-SM and CGSM system are dominated by the AHDs $D\left(\bar{\varsigma}_{p}\right)$. In fact, the AHD differences are mainly due to the different TAC sets. Through comparing the AHDs $D\left(\bar{\varsigma}_{p}\right)$ of the TAC set of the proposed SMx-SM with those of the C-GSM system, we can find the performances differences between them. Additionally, assuming that $\bar{\varsigma}_{1}<\bar{\varsigma}_{2}<\ldots<\bar{\varsigma}_{m}$, we have $F\left(\bar{\varsigma}_{1}\right)>F\left(\bar{\varsigma}_{2}\right)>\ldots>F\left(\bar{\varsigma}_{m}\right)$. As a result, a smaller $D\left(\bar{\varsigma}_{1}\right)$ results in a better BER performance. Table II presents the different AHDs for the $M=1$ aided proposed SMxSM and C-GSM systems having $N_{t}=8,16,32$ with $N_{u}=2$ and $N_{u}=4$, where only activated TACs convey information.

Observe from Table II that the AHDs $D\left(\bar{\varsigma}_{1}\right)$ of the SMxSM systems are smaller than those of the C-GSM systems for the above setups. As a result, the SMx-SM system is capable of exhibiting a better BER performance than the C-GSM system. The performance advantage becomes more dominant, as $N_{t}$ and $N_{u}$ increases.

\section{Performance Advantage over the V-BLAST system}

In this section, the performance advantage of the proposed SMx-SM system over the V-BLAST system is analyzed based on ABEP. The main difference between the proposed SMx-SM system and V-BLAST system lies in their transmit rates. Specifically, in the proposed $\mathrm{SMx}-$ SM system, SM is employed in each level, while only a APM symbol is employed in each level in V-BLAST system. Hence, with the same number of RF chains at the transmitter, the proposed SMx-SM system is capable of increasing the transmit rate significantly. Fig. 4 presents our transmit rate comparisons between the proposed QPSKaided SMx-SM system and the V-BLAST system for

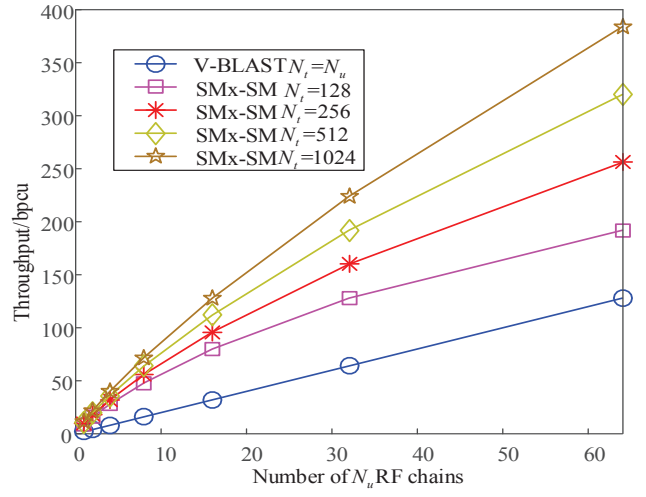

Fig. 4. Throughput comparisons for the SMx-SM and V-BLAST systems with same number of RF chains.

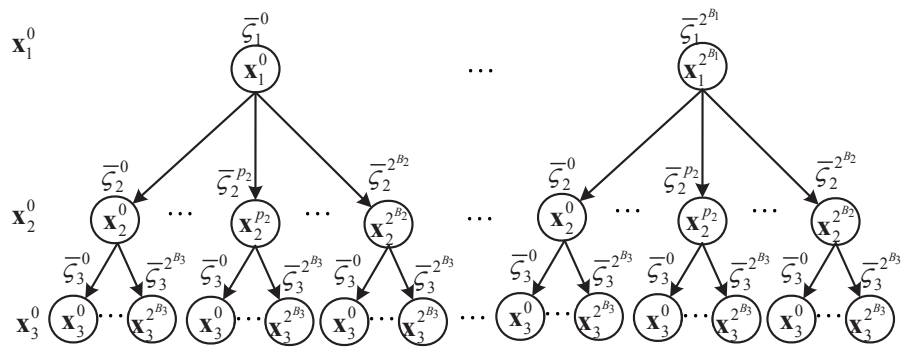

Fig. 5. The ABEP calculation of the $M=1$ aided SMx-SM system having $N_{u}=3$.

diverse numbers of RF chains spanning from $N_{u}=2$ to $N_{u}=64$, where bpcu denotes bits per channel use. Observe from Fig. 4, with the same constellation size and number of RF chains, the transmit rate of the proposed SMx-SM system increases, as $N_{t}$ increases and it is much larger than that of the V-BLAST system. For the fixed number of RF chains, the proposed SMx-SM system can employ lower-level modulation than V-BLAST system for achieving the same transmit rate. Observed from (13) and (14), with a lower modulation order, the SMx-SM system is able to obtain a larger value of $\bar{\zeta}$ by (13) and a smaller value of $F(\bar{\zeta})$ by (14), resulting in a smaller ABEP value. As a result, for a fixed number of RF chains and transmit rate, the SMx-SM system with $M=1$ is capable of achieving the maximum performance gain limit over the $\mathrm{V}$ BLAST system. For a high transmit rate $B$, it still remain difficult to compute the ABEP of SMx-SM system by (17). In order to obtain the theoretical maximum achievable performance advantage limit over the V-BLAST system, we further analyze the ABEP of the $M=1$ aided largescale SMx-SM system. For simplicity, we introduce the ABEP calculation of $N_{u}=3$ first, and then extend this process to larger values of $N_{u}$.

Case 1: $N_{u}=3$ and $M=1$ : For the case of $N_{u}=3$, 


$$
\begin{aligned}
& D\left(\bar{\varsigma}_{1}\right)=\frac{1}{B}\left(\sum_{p_{1}=1}^{\omega_{1}} d_{1}^{p_{1}}+\sum_{p_{2}=1}^{\omega_{2}} d_{2}^{p_{2}}+\sum_{p_{3}=1}^{\omega_{3}} d_{3}^{p_{3}}\right)=\frac{1}{B}\left(d_{1}^{p_{1} \neq 0}+d_{2}^{p_{2} \neq 0}+d_{3}^{p_{3} \neq 0}\right) \\
& D\left(\bar{\varsigma}_{2}\right)=\frac{1}{B}\left(\sum_{p_{2}=1}^{\omega_{2}} \sum_{p_{3}=1}^{\omega_{3}}\left(d_{2}^{p_{2}}+d_{3}^{p_{3}}\right)+\sum_{p_{1}=1}^{\omega_{1}} \sum_{p_{2}=1}^{\omega_{2}}\left(d_{1}^{p_{1}}+d_{2}^{p_{2}}\right)+\sum_{p_{1}=1}^{\omega_{1}} \sum_{p_{3}=1}^{\omega_{3}}\left(d_{1}^{p_{1}}+d_{3}^{p_{3}}\right)\right) \\
& \left.\left.=\frac{1}{B}\left(\sum_{p_{2}=1}^{\omega_{2}} \omega_{3} d_{2}^{p_{2}}+d_{3}^{p_{3} \neq 0}\right)+\sum_{p_{1}=1}^{\omega_{1}} \omega_{2} d_{1}^{p_{1}}+d_{2}^{p_{2} \neq 0}\right)+\sum_{p_{1}=1}^{\omega_{1}} \omega_{3} d_{1}^{p_{1}}+d_{3}^{p_{3} \neq 0}\right) \\
& =\frac{1}{B}\left(\left(\omega_{2}+\omega_{3}\right) d_{1}^{p_{1} \neq 0}+\left(\omega_{1}+\omega_{3}\right) d_{2}^{p_{2} \neq 0}+\left(\omega_{1}+\omega_{2}\right) d_{3}^{p_{3} \neq 0}\right) \\
& D\left(\bar{\varsigma}_{3}\right)=\frac{\sum_{p_{1}=1}^{\omega_{2}} \sum_{p_{2}=1}^{\omega_{p_{3}=1}} \sum_{3}^{\omega_{3}}\left(d_{1}^{p_{1}}+d_{2}^{p_{2}}+d_{3}^{p_{3}}\right)}{B}=\frac{1}{B}\left(\left(\omega_{2} \omega_{3}\right) d_{1}^{p_{1} \neq 0}+\left(\omega_{1} \omega_{3}\right) d_{2}^{p_{2} \neq 0}+\left(\omega_{1} \omega_{2}\right) d_{3}^{p_{3} \neq 0}\right)
\end{aligned}
$$

the SMx-SM system has three levels. Each level has a SM symbol set as $\mathbb{X}_{t}=\left[\mathbb{X}_{t}^{0}, \mathbb{X}_{t}^{1}, \ldots, \mathbb{X}_{t}^{p_{t}}, \ldots, \mathbb{X}_{t}^{2^{B_{t}}-1}\right] t=(1,2,3)$. Assuming that the first SMx-SM symbol carrying $2^{B}$ zeros is $\mathbf{x}^{1}=\left[\mathbf{x}_{1}^{0}, \mathbf{x}_{2}^{0}, \mathbf{x}_{3}^{0}\right]$ and the $j$-th $j=1, \ldots, 2^{B}$ SMx-SM symbol is $\mathbf{x}^{j}=\left[\mathbf{x}_{1}^{p_{1}}, \mathbf{x}_{2}^{p_{2}}, \mathbf{x}_{3}^{p_{3}}\right] p_{t} \in\left(0,2^{B_{t}}-1\right)$, the ABEP calculation of the SMx-SM system is depicted in Fig. 5 . Based on Fig. 5, (10) and (17), the ABEP can be expressed as

$$
P_{b}=\sum_{p_{1}=0}^{2^{B_{1}}-1} \sum_{p_{2}=0}^{2^{B_{2}}-1} \sum_{p_{3}=0}^{2^{B_{3}}-1}\left(d_{1}^{p_{1}}+d_{2}^{p_{2}}+d_{3}^{p_{3}}\right) F\left(\bar{\varsigma}=\bar{\varsigma}_{1}^{p_{1}}+\bar{\varsigma}_{2}^{p_{2}}+\bar{\varsigma}_{3}^{p_{3}}\right)
$$

where $d_{t}^{p_{t}}$ presents the HD between $\mathbf{x}_{t}^{p_{t}}$ and $\mathbf{x}_{t}^{0}$, which can be computed by

$$
\begin{aligned}
& d_{t}^{p_{t}}=\operatorname{Diff}\left(\mathbf{b}_{t}^{p_{t}}, \mathbf{b}_{t}^{0}\right) \in\left(0, B_{t}\right) \\
& \mathbf{b}_{t}^{p_{t}}=\operatorname{de} 2 \operatorname{bi}\left(p_{t}, B_{t}\right), t=(1,2,3)
\end{aligned}
$$

where $\operatorname{Diff}(x, y)$ is a function of returning difference between $x$ and $y$. Moreover, according to (13), $\bar{\varsigma}_{t}^{p_{t}}$ equals to

$$
\bar{\varsigma}_{t}^{p_{t}}=\frac{\left\|\mathbf{x}_{t}^{p_{t}}-\mathbf{x}_{t}^{0}\right\|^{2}}{\sigma^{2}}=\left\{\begin{array}{l}
0, p_{t}=0 \\
\frac{1}{\sigma^{2}}, p_{t} \neq 0 .
\end{array}\right.
$$

For the case of $N_{u}=3$ and $M=1$, there are three different values $\bar{\varsigma}$ as

$$
\begin{aligned}
& \bar{\varsigma}_{1}=\bar{\varsigma}_{1}^{p_{1}}+\bar{\varsigma}_{2}^{p_{2}}+\bar{\varsigma}_{3}^{p_{3}}=1 / \sigma^{2} \rightarrow\left\{\begin{array}{l}
p_{1}=p_{2}=0, p_{3} \neq 0 \\
p_{1}=p_{3}=0, p_{2} \neq 0 \\
p_{2}=p_{3}=0, p_{1} \neq 0
\end{array}\right. \\
& \bar{\varsigma}_{2}=\bar{\varsigma}_{1}^{p_{1}}+\bar{\varsigma}_{2}^{p_{2}}+\bar{\varsigma}_{3}^{p_{3}}=2 / \sigma^{2} \neq 0, p_{3}=0 \\
& \bar{\varsigma}_{3}=\bar{\varsigma}_{1}^{p_{1}}+\bar{\varsigma}_{2}^{p_{2}}+\bar{\varsigma}_{3}^{p_{3}}=3 / \sigma^{2} \rightarrow\left\{\begin{array}{l}
p_{1}, p_{3} \neq 0, p_{2}=0 \\
p_{2}, p_{3} \neq 0, p_{1}=0 \\
p_{2}, p_{3} \neq 0 .
\end{array}\right.
\end{aligned}
$$

The associated AHD can be computed as (25) with

$$
\begin{gathered}
\omega_{1}=2^{B_{1}}-1, \omega_{2}=2^{B_{2}}-1, \omega_{3}=2^{B_{3}}-1 \\
d_{t}^{p_{t} \neq 0}=\sum_{p_{t}=1}^{2^{B_{t}}-1} d_{t}^{p_{t}}=\sum_{u=1}^{B_{t}} u C_{B_{t}}^{u} .
\end{gathered}
$$

According to (17) and (25), the final ABEP for $N_{u}=3$ and $M=1$ is expressed as

$$
P_{b}=D\left(\bar{\varsigma}_{1}\right) F\left(\bar{\varsigma}_{1}\right)+D\left(\bar{\varsigma}_{2}\right) F\left(\bar{\varsigma}_{2}\right)++D\left(\bar{\varsigma}_{3}\right) F\left(\bar{\varsigma}_{3}\right),
$$

where $D\left(\bar{\varsigma}_{1}\right), D\left(\bar{\varsigma}_{2}\right)$ and $D\left(\bar{\varsigma}_{3}\right)$ can be computed by $(25)$.

Case 2: $N_{u}>\mathbf{3}, M=1$ : Based on the analysis of $N_{u}=3$ and $M=1$, it becomes clear that there are a total of $N_{u}$ different values of $\bar{\varsigma}=\bar{\varsigma}_{1}^{p_{1}}+\bar{\varsigma}_{2}^{p_{2}}+\ldots+\bar{\varsigma}_{N_{u}}^{p_{N_{u}}}$, which are associated with the number of non-zeros in $\left(p_{1}, p_{2}, p_{3}, \ldots, p_{N_{u}}\right)$. If the number of non-zeros in $\left(p_{1}, p_{2}, p_{3}, \ldots, p_{N_{u}}\right)$ is $r$, the values of $\bar{\varsigma}_{r}$ is computed by $(23)$ as

$$
\bar{\varsigma}_{r}=r / \sigma^{2} \text {. }
$$

Following this, the AHD of $D\left(\bar{\varsigma}_{r}\right)$ is computed. Owing to the $r$ non-zeros in $\left(p_{1}, p_{2}, p_{3}, \ldots, p_{N_{u}}\right)$, there are a total number of $C_{N_{u}}^{r}$ scenarios. For each scenario, the $r$ nonzeros are $\left(p_{k_{1}}, p_{k_{2}}, \ldots, p_{k_{r}}\right)$ for $k=1, \ldots, C_{N_{u}}^{r}$, hence the corresponding AHD can be computed as

$$
\begin{aligned}
& D\left(\bar{\varsigma}_{r}\right)=\frac{1}{B} \sum_{k=1}^{C_{N_{u}}^{r}}\left(\sum_{p_{k_{1}}=1}^{2^{B_{k_{1}}-1}} \cdots \sum_{p_{k_{r}}=1}^{2^{B_{k_{r}}-1}}\left(d_{k_{1}}^{p_{k_{1}}}+\cdots+d_{k_{r}}^{p_{k_{r}}}\right)\right) \\
& =\frac{1}{B} \sum_{k=1}^{C_{N_{u}}^{r}}\left(\prod_{v=k_{2}}^{k_{r}}\left(2^{B_{v}}-1\right) d_{k_{1}}^{p_{k_{1}} \neq 0}+\cdots+\prod_{v=k_{1},}^{k_{r}-1}\left(2^{B_{v}}-1\right) d_{k_{r}}^{p_{k_{r}} \neq 0}\right) \\
& =\frac{1}{B} \sum_{k=1}^{C_{N u}^{r}} \sum_{u=k_{1}}^{k_{r}}\left(\prod_{v=k_{1}, v \neq u}^{k_{r}}\left(2^{B_{v}}-1\right) d_{u}^{p_{u} \neq 0}\right),
\end{aligned}
$$

where $d_{u}^{p_{u} \neq 0}$ can be computed by (26). The final ABEP of the proposed SMx-SM with $M=1$ is expressed as

$$
\begin{aligned}
& P_{b}=\sum_{r=1}^{N_{u}} D\left(\bar{\varsigma}_{r}\right) F\left(\bar{\varsigma}_{t}=\frac{r}{\sigma^{2}}\right) \\
& =\frac{1}{B} \sum_{r=1}^{N_{u}}\left(\sum_{k=1}^{C_{N_{u}}^{r}} \sum_{u=k_{1}}^{k_{r}}\left(\prod_{v=k_{1}, v \neq u}^{k_{r}}\left(2^{B_{v}}-1\right) d_{u}^{p_{u} \neq 0}\right)\right) F\left(\bar{\zeta}_{r}=\frac{r}{\sigma^{2}}\right) .
\end{aligned}
$$

In summary, for a fixed number of RF chains and transmit rate, the theoretical maximum achievable performance advantage of the SMx-SM system over the V-BLAST system can be explicitly evaluated from (30).

\section{Compressive Sensing Based Detector for the Proposed SMX-SM System}

Although the SMx-SM system can be readily extended to large-scale MIMO systems, the ML detector's complexity becomes extremely high for large $N_{t}$ and $N_{u}$. Due to the sparsity of the SMx-SM symbol, a novel T-CS detector is designed for the SMx-SM system based on [27] by exploiting its sparsity. Specifically, the detection consists of three steps. First, we obtain a legitimate TAC $\hat{I}$ and the corresponding symbol vector $\hat{\mathbf{s}}$ using Orthogonal Matching Pursuit (OMP) algorithms [23]. Secondly, the estimated signal $(\hat{I}, \hat{\mathbf{s}})$ is judged reliable or unreliable by comparing it to a preset threshold. If the estimated signal is judged reliable, then it is deemed to be the final result. Otherwise, we design a new ordered TAC set. Finally, we estimate the transmit signal based on the new ordered TAC set. The main differences between the proposed T-CS and the Efficient CS (CS) detector of [27] is in the first step and in the TAC order design. The proposed T-CS detector operates as follows.

Step 1: Obtain the TAC $\hat{I}=\left(l_{1}, \ldots, l_{N_{u}}\right)$ and the corresponding symbol vector $\hat{\mathbf{s}}=\left(s_{1}, s_{2}, \ldots, s_{N_{u}}\right)$ via $\mathrm{OMP}$ algorithm and the structure of SMx-SM symbol. 
Specifically, the first ordered TA indices $\mathbf{l}_{1}$ can be obtained as

$$
\mathbf{l}_{1}=\left[l_{1}^{1}, l_{2}^{1}, \ldots, l_{N_{t}}^{1}\right]=\arg \operatorname{sort}\left(\left|\mathbf{H}^{H} \mathbf{y}\right|\right) .
$$

In the first step, in general, we have $\Lambda_{1}=l_{1}^{1}$. Then the APM symbol $s_{1}$ transmitted by this TA can be estimated as

$$
\tilde{s}_{1}=\mathbb{D}\left[\left(\mathbf{H}_{\Lambda_{1}}^{H} \mathbf{H}_{\Lambda_{1}}\right)^{-1} \mathbf{H}_{\Lambda_{1}}^{H} \mathbf{y}\right],
$$

where $\mathbb{D}(x)$ is defined as the digital demodulation function. After obtaining the residual $\mathbf{r}_{1}=\mathbf{y}-\mathbf{H}_{\Lambda_{1}} \tilde{s}_{1}$, a new set of ordered TA indices $\mathbf{l}_{2}$ can be obtained as

$$
\mathbf{l}_{2}=\left(l_{1}^{2}, l_{2}^{2} \ldots, l_{N_{t}}^{2}\right)=\arg \operatorname{sort}\left(\left|\mathbf{H}^{H} \mathbf{r}_{1}\right|\right) .
$$

In our SMx-SM system, the distribution of the activated TA index is very special. From the second step, in order to ensure that the index set obtained via the OMP algorithm in each step is legitimate, a decision process is required for determining whether or not the index set obtained is within the TAC set I. Specifically, for each step, we have to estimate one activate TA index for one SM symbol. In the second step, if the estimated $\Lambda_{1}=l_{1}^{1}$ in the first step is the activated TA index of the $i_{1}$-th SM symbol, then we do not have to estimate the $i_{1}$-th SM symbol's TA index again, all the possible TA indices $\lambda_{1}=\left[\sum_{j=1}^{i_{1}-1} N_{s m}^{j}+1, \sum_{j=1}^{i_{1}-1} N_{s m}^{j}+\right.$ $\left.2, \ldots, \sum_{j=1}^{i_{1}-1} N_{s m}^{j}+N_{s m}^{i_{1}}\right]$ of this $i_{1}$-th SM should be removed from $\mathbf{l}_{2}$ as $\mathbf{l}_{2}=\mathbf{l}_{2} \backslash \lambda_{1}=\left(l_{1}^{2}, \ldots, l_{N_{t}-N_{s m}^{i_{1}}}^{2}\right)$. After obtaining the new TA index vector $\mathbf{l}_{2}$, we get the estimated TA indices in the second step as $\Lambda_{2}=\left[\Lambda_{1}, l_{1}^{2}\right]$. Then the APM symbols transmitted by the index set $\Lambda_{2}$ are estimated as

$$
\tilde{\mathbf{s}}_{2}=\mathbb{D}\left[\left(\mathbf{H}_{\Lambda_{2}}^{H} \mathbf{H}_{\Lambda_{2}}\right)^{-1} \mathbf{H}_{\Lambda_{2}}^{H} \mathbf{y}\right] .
$$

After that, the residual $\mathbf{r}_{2}$ can be obtained as $\mathbf{r}_{2}=$ $\mathbf{y}-\mathbf{H}_{\Lambda_{2}} \tilde{\mathbf{s}}_{2}$. The above process continues, until we get the legitimate $\mathrm{TAC} \hat{I}=\left(l_{1}, \ldots, l_{N_{u}}\right)$. Then the corresponding symbol vector $\hat{\mathbf{s}}=\left(s_{1}, s_{2}, \ldots, s_{N_{u}}\right)$ can be estimated by minimum mean-squared error (MMSE) estimation as

$$
\hat{\mathbf{s}}=\mathbb{D}\left(\left[\left(\mathbf{H}_{\hat{I}}\right)^{H} \mathbf{H}_{\hat{I}}+\sigma^{2} \mathbf{I}_{N_{u}}\right]^{-1}\left(\mathbf{H}_{\hat{I}}\right)^{H} \mathbf{y}\right) .
$$

Taking $N_{t}=8, N_{u}=4, N_{s m}^{1}=N_{s m}^{2}=N_{s m}^{3}=N_{s m}^{4}=2$ for example, the OMP algorithm consists of four steps. In the first step, assuming that $\mathbf{l}_{1}=(4,6,8,5,3,2,7,1)$ we have $\Lambda_{1}=4$. In the second step, assuming that $\mathbf{l}_{2}=(3,5,8,6,4,2,7,1)$, we first obtain $\lambda_{1}=[3,4]$, and then remove the set $\lambda_{1}$ from $\mathbf{l}_{2}$ yielding $\mathbf{l}_{2}=(5,8,6,2,7,1)$. Hence we have $\Lambda_{2}=[4,5]$ in the second step. In the third step, assuming that $\mathbf{l}_{3}=(5,8,6,3,4,2,7,1)$, we first have $\lambda_{2}=[3,4,5,6]$, then we can obtain the set $\mathbf{l}_{3}=(8,2,7,1)$ and get $\Lambda_{3}=[4,5,8]$. In the fourth step, assuming that $\mathbf{l}_{4}=(5,2,1,3,4,8,7,6)$, we can have $\Lambda_{4}=[4,5,8,2]$. Finally, the estimated TAC is $\hat{I}=(4,5,8,2)$.

Step 2: Calculate the ED of the estimated signal $(\hat{I}, \tilde{\mathbf{s}})$ Step 3: If the above ED satisfies

$$
\left\|\mathbf{y}-\mathbf{H}_{\hat{I}} \tilde{\mathbf{s}}\right\|_{F}^{2} \leqslant V_{\mathrm{th}},
$$

where $V_{\mathrm{th}}=\beta N_{r} \sigma^{2}[27]$, then the output $(\hat{I}, \tilde{\mathbf{s}})$ is taken as the final detection result and $\beta$ is a constant.

Step 4: Otherwise, new ordered TAC indices are obtained using (31) as shown in Algorithm 1. The objective of

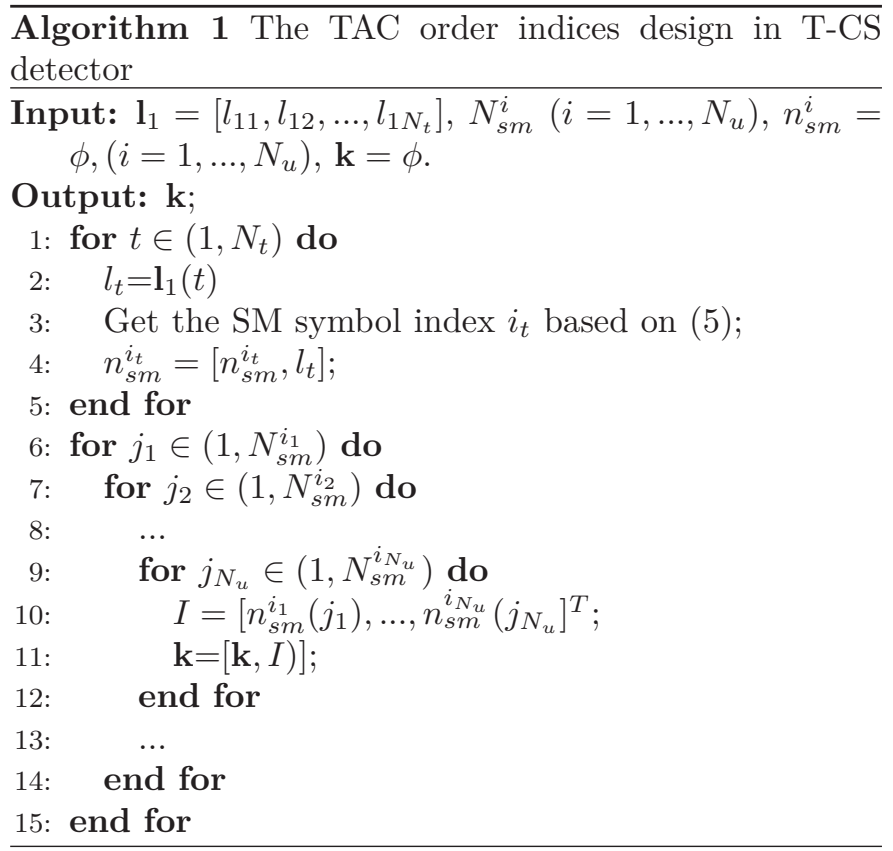

the TAC order design is to search for the highest probability TAC as soon as possible. Considering $N_{t}=8, N_{u}=4$, $N_{s m}^{1}=N_{s m}^{2}=N_{s m}^{3}=N_{s m}^{4}$ and $\mathbf{l}_{1}=(4,6,8,5,3,2,7,1)$ for example, we first get $n_{s m}^{i_{1}}=[4,3], n_{s m}^{i_{2}}=[6,5], n_{s m}^{i_{3}}=$ $[8,7], n_{s m}^{i_{4}}=[2,1]$. And the final order TAC can be expressed as

$$
\begin{aligned}
& \mathbf{k}=\left\{[4,6,8,2]^{T},[4,6,8,1]^{T},[4,6,7,2]^{T},[4,6,7,1]^{T},\right. \\
& {[4,5,8,2]^{T},[4,5,8,1]^{T},[4,5,7,2]^{T},[4,5,7,1]^{T}} \\
& {[3,6,8,2]^{T},[3,6,8,1]^{T},[3,6,7,2]^{T},[3,6,7,1]^{T}} \\
& \left.[3,5,8,2]^{T},[3,5,8,1]^{T},[3,5,7,2]^{T},[3,5,7,1]^{T}\right\} .
\end{aligned}
$$

For each TAC $I_{k_{i}}$, the corresponding symbol vector $\tilde{\mathbf{s}}_{k_{i}}$ is obtained via Eq. (35). The search terminates, whenever the output $\left(I_{k_{i}}, \tilde{\mathbf{s}}_{k_{i}}\right)$ satisfies

$$
\left\|\mathbf{y}-\mathbf{H}_{I_{k_{i}}} \tilde{\mathbf{s}}_{k_{i}}\right\|^{2} \leqslant V_{\mathrm{th}} .
$$

If no output $\left(I_{k_{i}}, \tilde{\mathbf{s}}_{k_{i}}\right)$ satisfies the above, the final result is deemed to be

$$
(\hat{I}, \hat{\mathbf{s}})=\underset{(I, \mathbf{s}) \in \mathbb{A}_{S M x-S M}}{\arg \min }\left\|\mathbf{y}-\mathbf{H}_{I} \mathbf{s}\right\|_{F}^{2},
$$

where $\mathbb{A}_{\mathrm{SMx}-\mathrm{SM}}=\left(\left(I_{k_{1}}, \tilde{\mathbf{s}}_{k_{1}}\right) \ldots,\left(I_{k_{N_{P}}}, \tilde{\mathbf{s}}_{k_{N_{P}}}\right)\right)$. After obtaining the combination of $(\hat{I}, \hat{\mathbf{s}})$, the information bits can be recovered by bit-to-symbol demapping principle.

\section{Vi. Message Passing Based Detector for the Proposed SMX-SM System}

\section{A. MP based Detector}

Although the T-CS detector constitutes an effective technique of detecting the $\mathrm{SMx}-\mathrm{SM}$ symbol, it still requires an exhaustive search through the TAC set to get the most likely signal, when the estimated signal is judged unreliable. As $N_{u}$ increases, the size of the TAC set increases significantly, hence making the exhaustive search impractical. In order to address this issue, a message passing based detector is developed for the proposed SMxSM system. In the SMx-SM system, each SMx-SM symbol 
consists of $N_{u}$ SM symbols, so that each SM symbol can be considered as a node and estimated separately. Specifically, according to (6), the receive signal of the $j$-th $\left(j=1, \ldots, N_{r}\right)$ antenna can be expressed as

$$
y_{j}=\mathbf{h}_{j,[i]} \mathbf{x}_{i}+\underbrace{\sum_{m=1, m \neq i}^{N_{u}} \mathbf{h}_{j,[m]} \mathbf{x}_{m}+n_{j}}_{N_{j, i}},
$$

where $\mathbf{x}_{i}\left(i=1, \ldots, N_{u}\right)$ denotes the $i$-th SM symbol having a length of $N_{s m}^{i}$ and $\mathbf{h}_{j,[i]}$ is a row vector of length $N_{s m}^{i}$, corresponding to $\mathbf{x}_{i}$. The term $N_{j, i}$ is approximated by a Gaussian random variable having a mean $u_{j i}$ and variance of $\sigma_{j i}^{2}$, which are given by

$$
\begin{gathered}
u_{j i}=E\left(\sum_{m=1, m \neq i}^{N_{u}} \mathbf{h}_{j,[m]} \mathbf{x}_{m}+n_{j}\right) \\
=\sum_{m=1, m \neq i}^{N_{u}} \sum_{\mathbf{s}_{m} \in \mathbb{X}_{s m}^{m}} \mathbf{h}_{j,[m]} p_{j m}\left(\mathbf{s}_{m}\right) \mathbf{s}_{m} \\
\sigma_{j i}^{2}=\operatorname{var}\left(\sum_{m=1, m \neq i}^{N_{u}} \mathbf{h}_{j,[m]} \mathbf{x}_{m}+n_{j}\right) \\
=\sum_{m=1, m \neq i}^{N_{u}} \sum_{\mathbf{s}_{m} \in \mathbb{X}_{s m}^{m}} p_{j m}\left(\mathbf{s}_{m}\right) \mathbf{h}_{j,[m]} \mathbf{s}_{m}\left(\mathbf{h}_{j,[m]} \mathbf{s}_{m}\right)^{H}- \\
\sum_{m=1, m \neq i}^{N_{u}}\left|\sum_{\mathbf{s}_{m} \in \mathbb{X}_{s m}^{m}} \mathbf{h}_{j,[m]} p_{j m}\left(\mathbf{s}_{m}\right) \mathbf{s}_{m}\right|^{2}+\sigma^{2}
\end{gathered}
$$

where $p_{j i}\left(\mathbf{s}_{i}\right)\left(i=1, \ldots, N_{u}\right)$ is the a posteriori probability. The value of $p_{j i}\left(\mathbf{s}_{i}\right)$ is initialized as $1 / \mathbb{X}_{s m}^{i}$ and then computed by

$$
p_{j i}\left(\mathbf{s}_{i}\right) \propto \prod_{n=1, n \neq j}^{N_{r}} \exp \left(\frac{-\left|y_{n}-u_{n i}-\mathbf{h}_{n,[i]} \mathbf{s}_{i}\right|}{2 \sigma_{n i}^{2}}\right) .
$$

The value of $p_{j i}\left(\mathbf{s}_{i}\right)$ is continuously updated. At the end of iterations, the $i$-th SM symbol can be estimated as

$$
\hat{\mathbf{x}}_{i}=\underset{\mathbf{s}_{i} \in \mathbb{X}_{s m}^{i}}{\arg \max } \prod_{n=1}^{N_{r}} \exp \left(\frac{-\left|y_{n}-u_{n i}-\mathbf{h}_{n,[i]} \mathbf{s}_{i}\right|}{2 \sigma_{n i}^{2}}\right) .
$$

In short, the MP-based detector designed for our SMx-SM system is given in Algorithm 2, where $N_{\text {iter }}$ is the number of iterations and $\alpha \in[0,1]$ is a damping factor.

\section{B. Complexity Analysis}

In this subsection, the complexity of the ML, of the TCS based and of the MP based detectors designed for our SMx-SM system is analyzed in terms of the numbers of real-valued multiplications and additions. For the specific matrices of $\mathbf{A} \in \mathbb{C}^{m \times n}, \mathbf{B} \in \mathbb{C}^{n \times p}, \mathbf{c} \in \mathbb{C}^{n \times 1}$ and $\mathbf{d} \in \mathbb{C}^{n \times 1}$, the operations of $\mathbf{A B},\|\mathbf{c}\|_{F}^{2}$ and $\mathbf{c} \pm \mathbf{d}$ require $(8 m n p-2 m p),(4 n-1)$ and $2 n$ Floating-point operations (Flops), respectively. Accordingly, the complexity orders of these detectors can be computed in detail as follows.

1) ML detector: The complexity order of the ML detector becomes

$$
C_{\mathrm{ML}}=\left(8 N_{r} N_{u}+4 N_{r}-1\right) 2^{B},
$$

because the operation $\left\|\mathbf{y}-\mathbf{H}_{I} \mathbf{s}\right\|_{F}^{2}$ in (7) requires $\left(8 N_{r} N_{u}+4 N_{r}-1\right)$ Flops and this operation is repeated $2^{B}$ times;

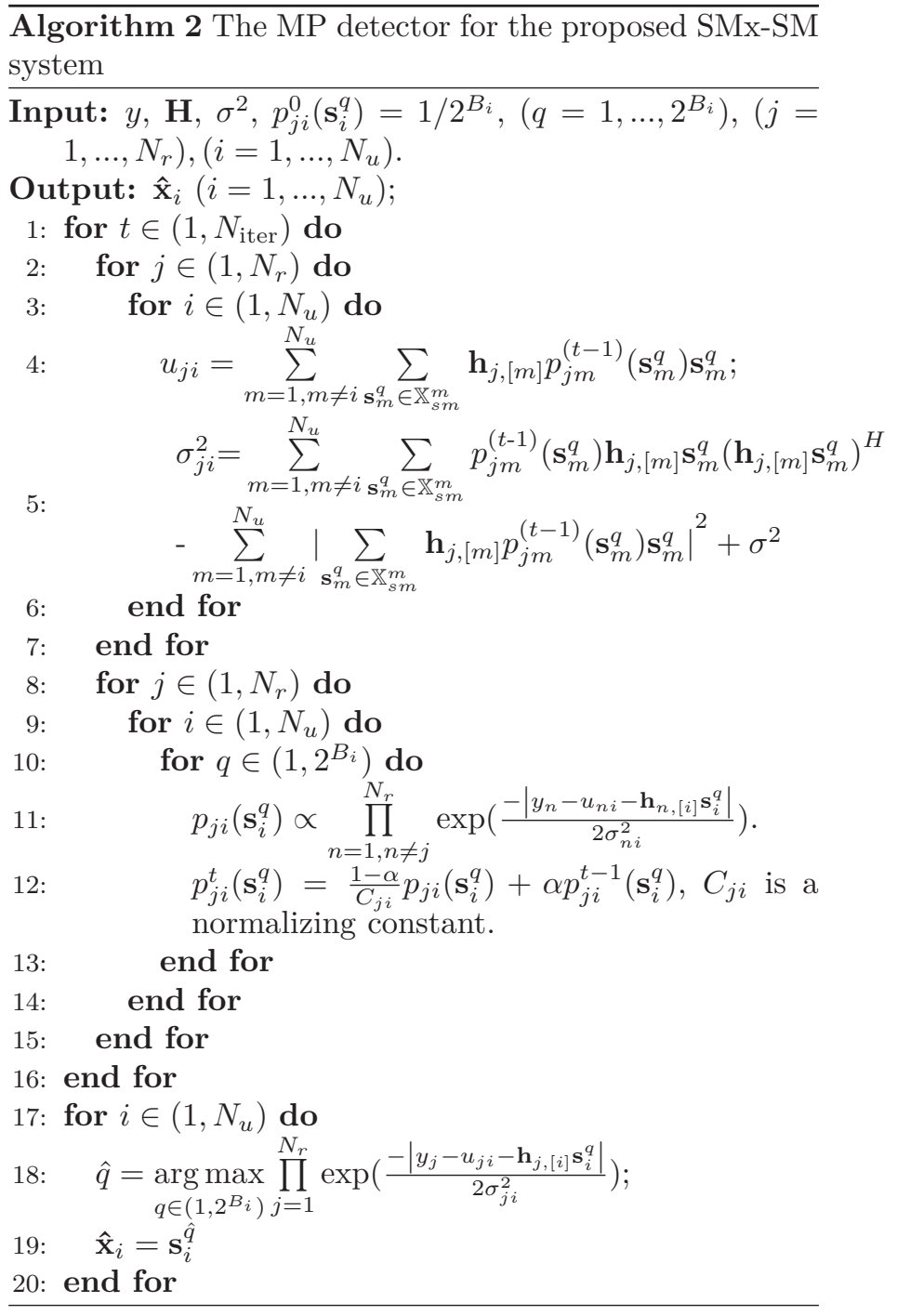

2) Proposed T-CS-based detector: The complexity order of the T-CS detector is

$$
\begin{aligned}
& C_{\mathrm{T}-\mathrm{CS}}=\sum_{t=1}^{N_{u}-1} 4 t^{3}+12 t^{2} N_{r}+7 t^{2}+6 N_{r} t+8 N_{r} t+8 N_{u} N_{r} N_{t} \\
& +2 N_{u} N_{t}+\left(4 N_{u}^{3}+12 N_{r} N_{u}^{2}+7 N_{u}^{2}+14 N_{r} N_{u}+4 N_{r}-1\right) \gamma_{1}
\end{aligned}
$$

where $\gamma_{1}$ is the average number of SMx-SM symbols. Specifically, the complexities of the proposed detectors consist of two parts: 1) the complexity of the OMP algorithm; 2) the complexity of $\left\|\mathbf{y}-\mathbf{H}_{I_{k_{j}}} \tilde{\mathbf{s}}_{k_{j}}\right\|_{F}^{2}$ associated with the specific number of $\gamma_{1}$. Firstly, the complexity order of the OMP algorithm is $N_{u}\left(8 N_{r} N_{t}+\right.$ $\left.2 N_{t}\right)+\sum_{t=1}^{N_{u}-1} 4 t^{3}+12 t^{2} N_{r}+7 t^{2}+6 N_{r} t+8 N_{r} t$, which can be inferred from Eqs. (31)-(35). For simplicity, the operations in (31) require $8 N_{r} N_{t}+2 N_{t}$ Flops and this operation is repeated $N_{u}$ times, while the operations in (32) and $\mathbf{r}_{t}=\mathbf{y}-\mathbf{H}_{\Lambda_{t}} \tilde{\mathbf{s}}_{t}$ require $4 t^{3}+12 t^{2} N_{r}+7 t^{2}+6 N_{r} t+8 N_{r} t$ Flops $t \in\left(1, N_{u}-1\right)$.

Secondly, if the SMx-SM symbol estimated by the OMP algorithm is unreliable, the operations in $\left\|\mathbf{y}-\mathbf{H}_{I_{k_{j}}} \tilde{\mathbf{s}}_{k_{j}}\right\|_{F}^{2}$ have been evaluated $\gamma_{1}$ times and each operation requires $\left(4 N_{u}^{3}+12 N_{r} N_{u}^{2}+7 N_{u}^{2}+14 N_{r} N_{u}+4 N_{r}-1\right)$ Flops.

2) Proposed MP-based detector: The complexity 


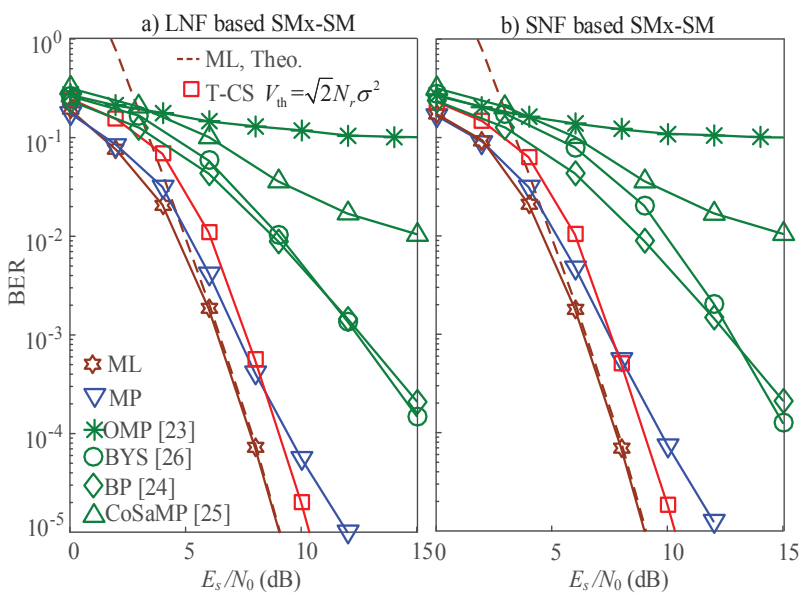

Fig. 6. Performances comparison with different detectors for $\mathrm{SMx}-$ SM systems having $N_{t}=32, N_{u}=4$ and $N_{r}=16$. a) LNFbased SMx-SM system having $\left(N_{s m}^{1}, N_{s m}^{2}, N_{s m}^{3}, N_{s m}^{4}\right)=(8,8,8,8)$ and $\left(M_{1}, M_{2}, M_{3}, M_{4}\right)=(2,2,2,2)$ at 16 bpcu b) SNF-based SMx-SM system having $\left(N_{s m}^{1}, N_{s m}^{2}, N_{s m}^{3}, N_{s m}^{4}\right)=(4,4,8,16)$ and $\left(M_{1}, M_{2}, M_{3}, M_{4}\right)=(4,4,2,1)$ at 16 bpcu.

\section{TABLE III}

COMPLEXITY COMPARISON OF DIFFERENT DETECTORS FOR SMX-SM SYSTEM

\begin{tabular}{|l|c|c|}
\hline \hline Scheme & Real-valued Flops & $\begin{array}{c}\text { Complexity normalized } \\
\text { by the ML detector }\end{array}$ \\
\hline OMP [23] & $3.32 \times 10^{4}$ & $0.04 \%$ \\
\hline BP $[24]$ & $1.19 \times 10^{5}$ & $0.16 \%$ \\
\hline CoSaMP $[25]$ & $2.55 \times 10^{5}$ & $0.34 \%$ \\
\hline BYS $[26]$ & $3.17 \times 10^{5}$ & $0.42 \%$ \\
\hline T-CS(SNF) & $1.5 \times 10^{6}$ & $2.06 \%$ \\
\hline T-CS(LNF) & $4.04 \times 10^{6}$ & $5.36 \%$ \\
\hline MP & $5.31 \times 10^{6}$ & $8.04 \%$ \\
\hline ML & $7.54 \times 10^{7}$ & $100 \%$ \\
\hline
\end{tabular}

order of the MP-based detector can be expressed as

$$
\begin{aligned}
& C_{\mathrm{MP}}=N_{\mathrm{iter}}\left[N_{r} \sum_{i=1}^{N_{u}} \sum_{m=1, m \neq i}^{N_{u}} 15 \cdot 2^{B_{m}}+N_{r} \sum_{i=1}^{N_{u}} 92^{B_{i}}\left(N_{r}-1\right)\right] \\
& +\sum_{i=1}^{N_{u}} 92^{B_{i}}
\end{aligned}
$$

Specifically, as seen from Algorithm 2, the complexity order of the MP detector is dominated by the operations in lines $4,5,11,12$ and 18, which require $N_{r} \sum_{i=1}^{N_{u}} \sum_{m=1, m \neq i}^{N_{u}} 8 \cdot 2^{B_{m}}, \quad N_{r} \sum_{i=1}^{N_{u}} \sum_{m=1, m \neq i}^{N_{u}} 7 \cdot 2^{B_{m}}$, $N_{r} \sum_{i=1}^{N_{u}} 92^{B_{i}}\left(N_{r}-1\right)$ and $\sum_{i=1}^{N_{u}} 92^{B_{i}}$ Flops, respectively.

\section{Simulation Results}

In this subsection, the performance results of the proposed SMx-SM associated with different antenna configurations are presented and compared. In all the simulation results, perfect channel state information is assumed.

\section{A. Performance vs Complexity Comparison between the Different Detectors of the SMx-SM System}

In this subsection, the performance and complexity of the different detectors of the LNF based and SNF based SMx-SM systems are compared in Fig. 6 and Table III. Both the SNF and LNF based SMx-SM systems have $N_{t}=32, N_{u}=4$ and $N_{r}=16$ at 16
TABLE IV

Complexity Comparison between SMx-SM system and C-GSM SYSTEM

\begin{tabular}{|c|c|c|c|c|}
\hline \hline & System & $\begin{array}{c}\text { Average } \\
\text { Search } \\
\text { Number }\end{array}$ & $\begin{array}{c}\text { Complexity } \\
\text { (Flops) }\end{array}$ & $\begin{array}{c}\text { Complexity } \\
\text { normalized } \\
\text { by the } \\
\text { C-GSM system }\end{array}$ \\
\hline$V_{\mathrm{th}=N_{r} \sigma^{2}}$ & $\begin{array}{c}\text { C-GSM } \\
\text { (ECS) }\end{array}$ & $1.52 \times 10^{4}$ & $4.0 \times 10^{7}$ & $100 \%$ \\
\cline { 2 - 5 } & $\begin{array}{c}\text { SMx-SM } \\
\text { (T-CS) }\end{array}$ & $3.80 \times 10^{3}$ & $1.04 \times 10^{7}$ & $26 \%$ \\
\hline \multirow{2}{*}{$V_{\mathrm{th}=\sqrt{2} N_{r} \sigma^{2}}$} & $\begin{array}{c}\text { C-GSM } \\
\text { (ECS) }\end{array}$ & $1.93 \times 10^{3}$ & $5.13 \times 10^{6}$ & $100 \%$ \\
\cline { 2 - 5 } & $\begin{array}{c}\text { SMx-SM } \\
\text { (T-CS) }\end{array}$ & 622 & $1.66 \times 10^{6}$ & $33 \%$ \\
\hline
\end{tabular}

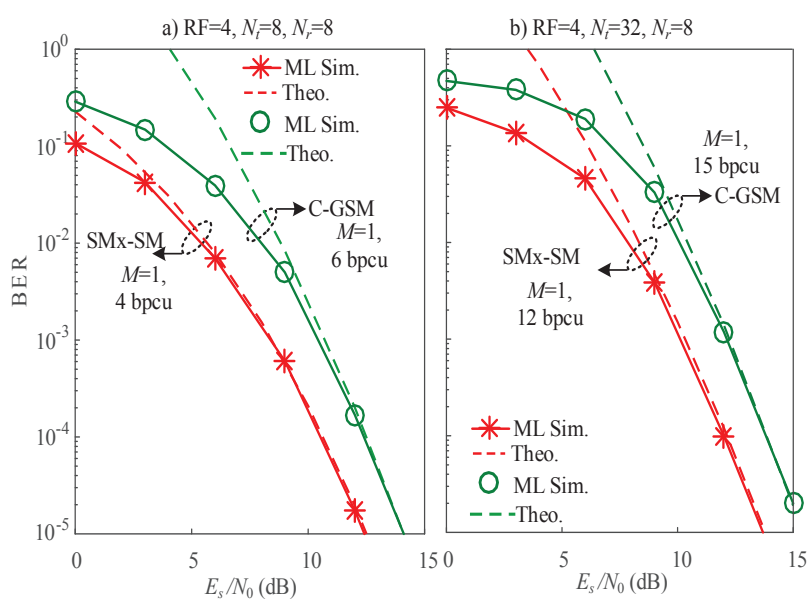

Fig. 7. Performance comparisons between $M=1$ aided SMx-SM and C-GSM systems having four RF chains. a) SMx-SM system having $N_{t}=8$ and $\left(N_{s m}^{1}, N_{s m}^{2}, N_{s m}^{3}, N_{s m}^{4}\right)=(2,2,2,2)$; b) SMx-SM system having $N_{t}=32$ and $\left(N_{s m}^{1}, N_{s m}^{2}, N_{s m}^{3}, N_{s m}^{4}\right)=(8,8,8,8)$.

bpcu. Specifically, $\left(N_{s m}^{1}, N_{s m}^{2}, N_{s m}^{3}, N_{s m}^{4}\right)=(8,8,8,8)$ and $\left(M_{1}, M_{2}, M_{3}, M_{4}\right)=(2,2,2,2)$ are employed in the LNFbased SMx-SM system, while $\left(N_{s m}^{1}, N_{s m}^{2}, N_{s m}^{3}, N_{s m}^{4}\right)=$ $(4,4,8,16)$ and $\left(M_{1}, M_{2}, M_{3}, M_{4}\right)=(4,4,2,1)$ are employed in the SNF-based SMx-SM system to achieve the same transmit rate of $16 \mathrm{bpcu}$. Moreover, the theoretical results are added as benchmarkers. Observe from Fig. 6 that the upper bound derived becomes very tight upon increasing the SNR values for the proposed SMx-SM system, which is helpful for evaluating the BER performances of the proposed SMx-SM system. Observed from Fig. 6 and Table III, the proposed T-CS and MP detectors are capable of reducing the complexity to about $10 \%$ of the ML detector at a modest performance erosion and also providing a significant performance gain over the conventional CS detectors at some extra complexity. The proposed LNF-based and SNF-based SMx-SM systems offer similar performances when employing the same detector. Since the total number of TACs of the SNF-based SMx-SM system is lower than that of its LNF-based counterpart, the SNFbased SMx-SM system exhibits a lower complexity than its LNF-based counterpart, when employing the same T-CS detector.

B. Performance Comparison Between the Proposed SMx$S M$ and the C-GSM System

Fig. 7 compares the performance attained by an $N_{u}=4$ RF chain based SMx-SM systems to those of C-GSM systems having $N_{t}=N_{r}=8$ and $N_{t}=32, N_{r}=8$. The main differences between the proposed SMx-SM system and the C- 


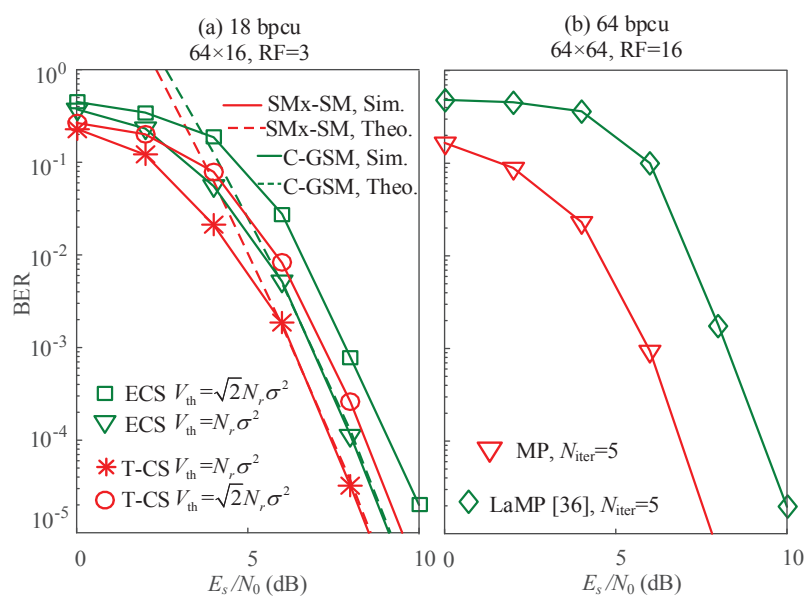

Fig. 8. Performance comparisons between QPSK aided SMx-SM and C-GSM systems having different antenna configurations. a) $N_{t}=64$ $N_{r}=16, N_{u}=3$ and $\left.\left(N_{s m}^{1}, N_{s m}^{2}, N_{s m}^{3}\right)=(16,16,32) ; \mathrm{b}\right) N_{t}=64$ $N_{r}=64, N_{u}=16$ and $\left(N_{s m}^{i}\right)=4, i=(1, \ldots, 16)$.

GSM system lies in their TAC sets, hence we employ $M=1$ and ML detectors in Fig. 7 for investigating the maximum performance gap between them. Since the throughput of the LNF-based SMx-SM system is higher than that of its SNF-based counterpart, we employ an LNF-based SMxSM system for comparison in the following figures. As predicted in Table II, the SMx-SM system having $N_{t}=8$ and $4 \mathrm{RF}$ chains at $4 \mathrm{bpcu}$ is capable of outperforming the C-GSM system having $N_{t}=8$ and $4 \mathrm{RF}$ chains at $6 \mathrm{bpcu}$ by $2 \mathrm{~dB}$ at $\mathrm{BER}=10^{-5}$. A similar trend can be observed for the case of $N_{t}=32, N_{r}=8$.

In order to provide further insights, Fig. 8 (a) and Table IV compare the performance and complexity of the SMxSM system to those of C-GSM system having $N_{t}=64$, $N_{r}=16$ and $N_{u}=3$ at the same transmit rate $18 \mathrm{bpcu}$. Specifically, For the case of $N_{t}=64, N_{r}=16$ and $N_{u}=3$ in Fig. 8 (a), the TAC set size of the C-GSM is $2^{15}$, BPSK is employed for the C-GSM system to achieve $18 \mathrm{bpcu}$, while the TAC set size of the LNF based SMx-SM system is $2^{13}$, whilst $\left(M_{1}, M_{2}, M_{3}\right)=(4,4,2)$ are employed to achieve $18 \mathrm{bpcu}$. In this case, $N_{u}$ is small, T-CS and ECS [27] detectors are employed for SMx-SM system and C-GSM system, respectively. Since the TAC size of the SMx-SM system is much smaller than that of the C-GSM system, the average number of search comparisons in the T-CS detector based SMx-SM system is much smaller than that of the ECS based C-GSM system, which results in a much lower complexity. As seen from Fig. 8(a) and Table IV, the SMx-SM system having $3 \mathrm{RF}$ chains with $V_{\mathrm{th}}=N_{r} \sigma^{2}$ and based on the T-CS detector is capable of providing around $1 \mathrm{~dB}$ performance gain over the $\mathrm{C}$ GSM system with $V_{\mathrm{th}}=N_{r} \sigma^{2}$ based on the ECS detector with a $74 \%$ complexity reduction. The same trend can be observed for the CS based detectors having $V_{\mathrm{th}}=\sqrt{2} N_{r} \sigma^{2}$.

Fig. 8 (b) compares the performance of the SMx-SM system to those of C-GSM system having $N_{t}=64, N_{r}=$ 64 and $N_{u}=16$ at $64 \mathrm{bpcu}$. In this case, the TAC set size of the C-GSM is $2^{48}$, BPSK is employed for the C-GSM system to achieve $64 \mathrm{bpcu}$, while the TAC set size of the LNF based SMx-SM system is $2^{32}$, QPSK $\left(M_{i}=4, i=\right.$ $(1, \ldots, 16))$ is employed to achieve 64 bpcu. Since the TAC set size is excessive, the threshold based CS detectors are no longer practical for the case of $N_{t}=64$ and $N_{u}=16$, so that MP detector is employed in Fig. 8 (b). As observed from Fig. 8 (b), the SMx-SM system with the proposed MP detector outperforms the C-GSM system with Layer MP (LaMP) detector in [37] by $2.5 \mathrm{~dB}$ at $\mathrm{BER}=10^{-5}$.

\section{Performance Comparison with the V-BLAST System}

Fig. 9 compares the performance results of the $M=1$ and LNF based SMx-SM and 16-QAM aided V-BLAST systems having $4 \mathrm{RF}$ chains associated with different values of $N_{r}$ at $16 \mathrm{bpcu}$. ML detectors are employed for the SMx-SM and V-BLAST system. Observe from Fig. 9 that for both cases of $N_{r}=8$ and $N_{r}=16$, the maximum performance gap between the SMx-SM system having 4 $\mathrm{RF}$ chains and V-BLAST system having $4 \mathrm{RF}$ chains at 16 bpcu is around $6 \mathrm{~dB}$.

Fig. 10 compares the performance results of the LNF based SMx-SM and V-BLAST systems having 8 RF chains at $32 \mathrm{bpcu}$ and having $16 \mathrm{RF}$ chains at $64 \mathrm{bpcu}$, respectively. For the case of $8 \mathrm{RF}$ chains, $N_{t}=64, M_{i}=$ $2,(i=1, \ldots, 8)$ and $N_{t}=128, M=1$ are employed in our SMx-SM system, while 16-QAM is employed in the VBLAST system. For the case of $16 \mathrm{RF}$ chains, $N_{t}=128$, $M_{i}=2,(i=1, \ldots, 8)$ and $N_{t}=256, M=1$ are employed in our SMx-SM system at 64bpcu, while 16-QAM is employed in the V-BLAST system. For the case of $16 \mathrm{RF}$ chains, the TAC set size of the C-GSM system having $N_{t}=128$ and $N_{t}=256$ are $2^{66}$ and $2^{83}$, hence TAC mapping and demapping become impractical. We also added the theoretical results of the $M=1$ based SMx-SM system calculated from (30) for the above antenna configurations as benchmarkers. Moreover, MP detectors are employed for both the SMx-SM and for the V-BLAST systems.

For the case of $8 \mathrm{RF}$ chains, observe in Fig. 10 (a) that the MP detector based SMx-SM systems having $N_{t}=$ $64, M_{i}=2,(i=1, \ldots, 8)$ and $N_{t}=128, M=1$ at $32 \mathrm{bpcu}$ offer a similar performance and approach the theoretical result of the SMx-SM system having $N_{t}=128, M=1$. It is also clear that the SMx-SM system having $8 \mathrm{RF}$ chains is capable of providing a $6 \mathrm{~dB}$ performance gain over the $\mathrm{V}$ BLAST system having the same number of RF chains and throughput of $32 \mathrm{bpcu}$. A similar trend can be observed for the case of $16 \mathrm{RF}$ chains in Fig . 10 (b). As for the complexity comparison associated with the fixed values of $N_{u}$ and $B$, the SMx-SM system using the MP detector offers the same complexity as the V-BLAST system in conjunction with the MP detector. This is because the term $\mathbf{s}_{m}$ in (41)-(44) only has a single non-zero element, hence the number of Flops in $\mathbf{h}_{j,[m]} p_{j m}\left(\mathbf{s}_{m}\right) \mathbf{s}_{m}$ using (41)(44) is the same as that of the V-BLAST system.

\section{Conclusions}

In this paper, a novel SMx-SM scheme was proposed for large-scale MIMO systems. We first propose a pair of mapping methods for the SMx-SM system, which exhibit a similar performance. Then we proposed a T-CS detector and MP detector for large-scale SMx-SM systems. Specifically, the T-CS detector is capable of approaching the ML detector's performance, despite its $90 \%$ complexity reduction. The T-CS detector is more suitable for largescale SMx-SM systems having small number of RF chains, 

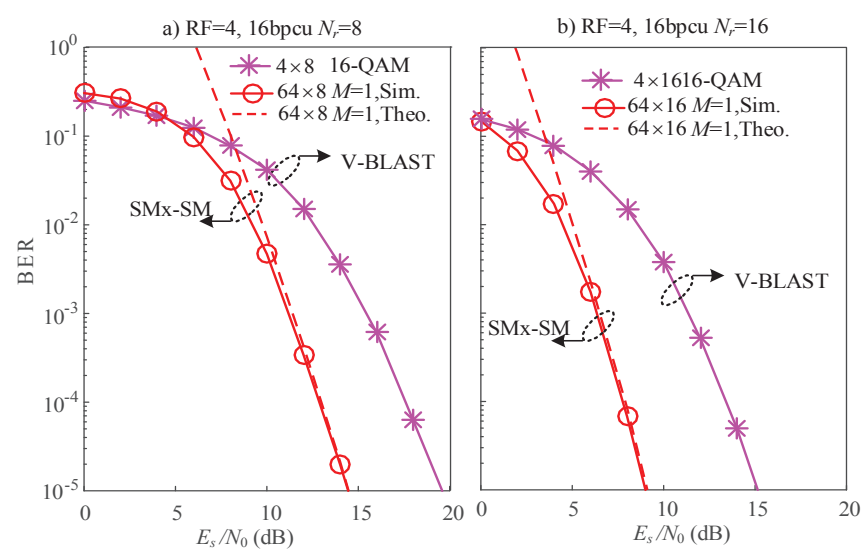

Fig. 9. Performance comparisons between $M=1$ aided SMx-SM and 16-QAM aided V-BLAST systems having four RF chains at $16 \mathrm{bpcu}$. a) $N_{r}=8$; b) $N_{r}=16$.

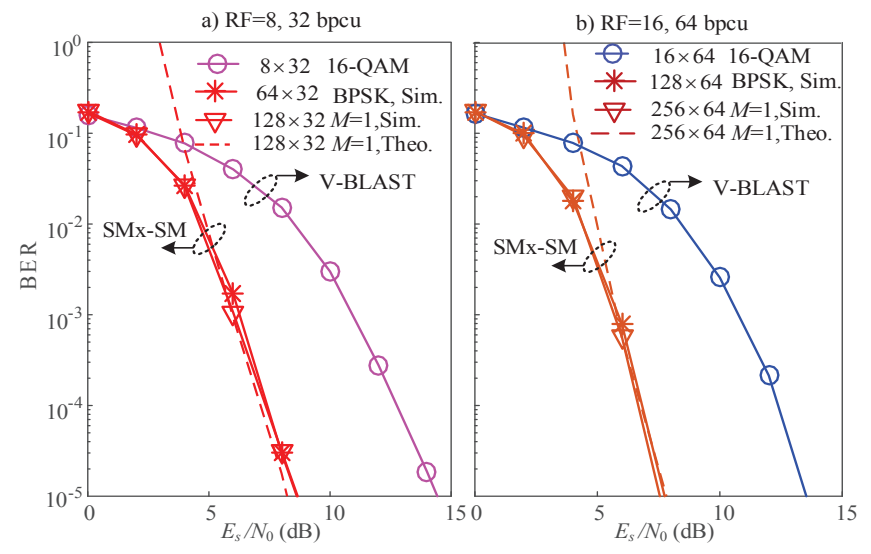

Fig. 10. Performance comparisons between SMx-SM and 16-QAM aided V-BLAST systems having $8 \mathrm{RF}$ chains and $16 \mathrm{RF}$ chains. a) 8 $\mathrm{RF}$ chains at $32 \mathrm{bpcu}$ b) $16 \mathrm{RF}$ chains at $64 \mathrm{bpcu}$.

while the MP detector is better for large-scale SMx-SM systems having a large number of RF chains. Compared to the conventional GSM system, the proposed SMx-SM system exhibits a better performance vs lower complexity trade-off, and is easier to extend to large-scale TAs having a large number of activated TAs. Moreover, the proposed SMx-SM system is capable of providing $6 \mathrm{~dB}$ performance gain over the classic VBLAST system with the same number of RF chains and throughput.

\section{REFERENCES}

[1] A. Chockalingam and B. S. Rajan, Large MIMO Systems. Cambridge, U.K.: Cambridge Univ. Press, 2014.

[2] E. Larsson, O. Edfors, F. Tufvesson, and T. Marzetta, "Massive MIMO for next generation wireless systems," IEEE Commun. Mag., vol. 52, no. 2, pp. 186-195, Feb. 2014.

[3] J. Andrews, S. Buzzi, W. Choi, S. Hanly, A. Lozano, A. Soong, and J. Zhang, "What Will 5G Be?" IEEE J. Sel. Areas Commun., vol. 32,no. 6, pp. 1065-1082, June 2014

[4] P. Wolniansky, G. Foschini, G. Golden, and R. Valenzuela, "VBLAST: anarchitecture for realizing very high data rates over the rich-scattering wireless channel," in Proc. 1998 International Symp. Signals, Syst., Electron., Pisa, Oct. 1998, pp. 295300.

[5] S. M. Alamouti, "A simple transmitter diversity scheme for wireless communications," IEEE J. Select. Areas Commun., vol. 16, no. 8, pp. 1451-1458, Oct. 1998.

[6] R. Mesleh, H. Haas, S. Sinanovic, C. W. Ahn, and S. Yun, "Spatial modulation," IEEE Trans. Veh. Technol., vol. 57, no 4, pp. 2228-2241, Jul. 2008.
[7] M. Di Renzo, H. Haas, A. Ghrayeb, S. Sugiura, and L. Hanzo, "Spatial modulation for generalized MIMO: challenges, opportunities and implementation," Proceedings of the IEEE, vol. 102, no. 1, pp. 56-103, Jan. 2014

[8] P. Yang, M. Di Renzo, Y. Xiao, S. Q. Li and L. Hanzo,"Design guidelines for spatial modulation," IEEE Commun. Surveys Tuts., vol. 17, no. 1, pp. 6-26, First Quart. 2015.

[9] N. Serafimovski, A. Younis, R. Mesleh, P. Chambers, M. D. Renzo, C. X. Wang, P. M. Grant, M. A. Beach and H. Haas, "Practical implementation of spatial modulation," IEEE Trans. Veh. Technol., vol. 62, no. 9, pp. 4511-4523, June, 2013.

[10] P. Yang, Y. Xiao, Y. L. Guan, et al, "Single-carrier spatial modulation: A promising design for large-scale broadband antenna systems," IEEE Commun. Surveys Tuts., vol. 18, no. 3, pp. 1687-1716, Feb. 2016

[11] S. Wang, Y. Li, M. Zhao, and J. Wang, "Energy efficient and low-complexity uplink transceiver for massive spatial modulation MIMO," IEEE Trans. Veh. Technol., vol. 64, no. 10, pp. 4617-4632, Oct. 2015

[12] S. Wang, Y. Li, and J. Wang, "Multiuser detection in massive spatial modulation MIMO with low-resolution ADCs," IEEE Trans. Wireless Commun., vol. 14, no. 4, pp. 2156-2168, Apr. 2015.

[13] L. He, J. Wang, J. Song, and L. Hanzo, "On the multi-user, multi-cell massive spatial modulation uplink: How many antennas for each user?,"IEEE Trans. Wireless Commun., vol. PP, no. 99, pp. 1-1, Dec. 2016.

[14] A. Younis, N. Serafimovski, R. Mesleh, and H. Haas, "Generalised spatial modulation," in Proc. 2010 Signals, Syt. Comput., Pacific Grove, Nov. 2010, pp. 1498-1502.

[15] J. L. Fu, C. P. Hou, W. Xiang, L. Yan, and Y. H. Hou, "Generalised spatial modulation with multiple active transmit antennas," in Proc. IEEE GLOBECOM Workshops, FL, Miami, Dec. 2010, pp. 839-844.

[16] R. M. Legnain, R. H. M. Hafez, I. Marsland and A. Legnain, " A novel spatial modulation using MIMO spatial multiplexing," in Proc. 2013 ICCSPA., Sharjash, United Arab Emirates, Feb. 2013, pp. 1-4.

[17] J. Wang, S. Jia, and J. Song, "Generalised spatial modulation with multiple active transmit antennas and low complexity detection scheme," IEEE Trans. Wireless Commun., vol. 11, no. 4, pp. 1605-1615, Apr. 2012.

[18] T. Datta and A. Chockalingam, "On generalized spatial modulation," in Proc. 2013 WCNC, Shanghai, China, April 2013, pp. 2716-2721.

[19] C. Lin, W. Wu, and C. Liu, "Low-complexity ML detectors for generalized spatial modulation systems,"IEEE Trans. Commun vol. 63, no. 11, pp. 4214-4230, Nov. 2015.

[20] J. Cal-Braz and R. Sampaio-Neto, "Nested maximum likelihood group detection in generalized spatial modulation MIMO systems," IEEE Commun. Lett., vol. 18, no. 6, pp. 953-956, June 2014.

[21] C. Chen, C. Li, and Y. Huang, "An improved ordered-block MMSE detector for generalized spatial modulation,"IEEE Commun. Lett., vol. 19, no. 5, pp. 707-710, May 2015.

[22] J. C. Braz and R. S. Neto, "Projection-based list detection in generalized spatial modulation MIMO systems,"IEEE Commun. Lett., vol. 19, no. 7, pp. 1145-1148, July 2015.

[23] C. Yu, S. Hsieh, H. Liang, C. Lu, W. Chung, S. Kuo and S. Pei, "Compressed sensing detector design for space shift keying in MIMO systems," IEEE Commun. Lett., vol. 16, no. 10, pp. 1556-1559, Oct. 2012.

[24] W. Liu, N. Wang, M. Jin, and H. Xu, "Denoising detection for the generalized spatial modulation system using sparse property," IEEE Commun. Lett., vol. 18, no. 1, pp. 22-25, Jan. 2014.

[25] A. G. Rodriguez and C. Masouros, "Low-complexity compressive sensing detection for spatial modulation in large-scale multiple access channels," IEEE Trans. Commun., vol. 63, no. 7 , pp. 2565-2579, July 2015.

[26] C. Yang, P. Cheng, Z. Chen, J. A. Zhang, Y. Xiao abd L. Gui, "Near-ML low-complexity detection for generalized spatial modulation", IEEE Commun. Lett., vol. 20, no. 3, pp. 618-621, Mar. 2016.

[27] L. Xiao, P. Yang, Y. Xiao, S. Fan, M. D. Renzo, W. Xiang, and S. $\mathrm{Li}$ "Efficient compressed sensing detectors for generalized spatial modulation systems," IEEE Trans. Veh. Technol., vol. pp, no. 99, pp. 1-14, April, 2016.

[28] L. Xiao, P. Yang, Y. Xiao, J. Liu, S. Fan, B. Dong, and S. Li, "An improved soft-input soft-output detector for generalized spatial modulation," IEEE Signal Proc. Lett., vol. 23, no. 1, pp. 30-34, Jan. 2016. 
[29] L. Xiao, L. Dan, Y. Zhang, Y. Xiao, P. Yang, and S. Li, "A lowcomplexity detection scheme for generalized spatial modulation aided single carrier systems," IEEE Commun. Lett., vol. 19, no. 6, pp. 1069-1072, June, 2015

[30] L. Xiao, P. Yang, Y. Zhao, Y. Xiao, J. Liu, and S. Li, "Lowcomplexity tree search-based detection algorithms for generalized spatial modulation aided single carrier systems," in Proc. IEEE 2016 ICC., Malaysia, May, 2016.

[31] S. Jin, W. Choi, J. Park, and D. Park, "Linear predecoding design for mutual information maximization in generailzed spatial modulation with finite alphabet inputs"IEEE Commun. Lett., vol. PP, no. 99, pp. 1-1, Aug. 2015.

[32] J. Freudenberger, and S. Shavgulidze, "Signal constellations based on eisenstein integers for generalized spatial modulation," IEEE Commun. Lett., vol. 4, no. 6, pp. 613-616, Nov., 2016.

[33] T. L. Narasimhan, P. Raviteja and A. Chockalingam"Generalized spatial modulation in large-scale multiuser MIMO systems," IEEE Trans. Wireless Commun. vol. 14, no. 7, pp. 1536-1276, March 2015.

[34] P. Patcharamannepakorn, S. Wu, C. Wang, E. M. Aggoune, M. M. Alwakeel, X. Ge, and M. Di. Renzo,"Spectral, energy, and economic efficiency of $5 \mathrm{G}$ multicell massive mimo systems with generalized spatial modulation", IEEE Trans. Veh. Technol., vol. 65 , no. 12, pp. 9715-9731, Dec., 2016

[35] N. Ishikawa, R. Rajashekar, S. Sugiura and L. Hanzo"Generalized spatial modulation based reduced-RFChain millimeter-wave communications," IEEE Trans. Veh. Technol., vol. PP, no. 99, pp. 1-1, April, 2016.
[36] A. I. Ibrahim, T. Kim and D. J. Love,"On the achievable rate of generalized spatial modulation using multiplexing under a Gaussian mixture model," IEEE Trans. commun., vol. 64, no. 4, pp. 1588-1599, Jan., 2016

[37] T. L. Narasimhan and A. Chockalingam, "On the capacity and performance of generalized spatial modulation"IEEE Commun. Lett., vol. 20, no. 2, pp. 252-255, Nov., 2015.

[38] Z. An, J. Wang, J. Wang, and J. Song, "Mutual information and error probability analysis on generalized spatial modulation system", IEEE Trans. Commun. vol. PP, no. 99, pp. 1-1, Dec. 2016.

[39] R. Mesleh, S. S. Ikki, and H. M. Aggoune, "Quadrature spatial modulation," IEEE Trans. Veh. Technol., vol. 64, no. 6, pp. 2738-2742, June. 2015.

[40] L. Xiao, P. Yang, S. Fan, S. Li, L. Song, and Y. Xiao, "Lowcomplexity signal detection for large-scale quadrature spatial modulation systems" IEEE Comun. Lett., vol. PP, no. 99, pp. 1-4, Aug., 2016.

[41] C.-C. Cheng, H. Sari, S. Sezginer and Y. Su, "Enhanced spatial modulation with multiple signal constellations,"IEEE Trans. Commun., vol. 63, no. 6, June 2015, pp. 2237-2248.

[42] C.-C. Cheng, H. Sari, S. Sezginer and Y. Su, "New signal designs for enhanced spatial modulation "IEEE Trans. Wireless Commun., vol. 15, no. 11, Nov. 2016, pp.7766-7777.

[43] O. Osman, "Variable active antenna spatial modulation,"IEEE IET Microwaves, Antennas Propagation vol. 9, no. 15, pp. 18161824 Oct. 2015. 2237-48. 